\title{
Development of indanones and isatins as non-cytotoxic inhibitors of cholinesterases
}

\author{
Josephine M. Gießel ${ }^{1}$, Anne Loesche ${ }^{1}$, Sophie Hoenke ${ }^{1}$, Immo Serbian ${ }^{1}$, Ahmed Al-Harrasi ${ }^{2}$ \\ and René Csuk ${ }^{1, *}$ \\ ${ }^{1}$ Full Address: Martin-Luther-University Halle-Wittenberg, Organic Chemistry, Kurt-Mothes-Str. 2, D-06120 \\ Halle (Saale), Germany \\ 2 Full Address: University of Nizwa, Chair of Oman's Medicinal Plants and Marine Natural Products, \\ P.O. Box 33, PC 616, Birkat Al-Mauz, Nizwa, Sultanate of Oman
}

\begin{abstract}
A small library of indanone-amides and substituted isatin derivatives has been prepared; these compounds have been investigated for their ability to act as inhibitors for the enzymes acetyl- and butyrylcholinesterase (AChE, BChE). Several of them were moderate inhibitors for AChE and not cytotoxic for a variety of human tumor cell lines as well as for non-malignant mouse fibroblasts. In this library consisting of 49 derivatives, 5,7-dibromo-4-iodoisatin was shown to be a good mixed-type inhibitor for $\mathrm{AChE}\left(\mathrm{K}_{\mathrm{i}}=2.52 \pm 0.61\right.$ $\mu \mathrm{M}$ and $\left.\mathrm{K}_{\mathrm{i}^{\prime}}=11.74 \pm 1.31 \mu \mathrm{M}\right)$ but this compound also acted as a dual inhibitor for $\mathrm{BChE}\left(\mathrm{K}_{\mathrm{i}}=4.49 \pm 0.32 \mu \mathrm{M}\right.$ and $\left.\mathrm{K}_{\mathrm{i}^{\prime}}=6.56 \pm 0.57 \mu \mathrm{M}\right)$. Interestingly, $N$-hexyl-1-oxo-2,3-dihydro-1H-indene-2-carboxamide was cytotoxic especially for MCF-7 breast adenocarcinoma cells $\left(\mathrm{EC}_{50}=4.28 \pm 0.5 \mu \mathrm{M}\right)$.
\end{abstract}

Keywords: acetylcholinesterase; butyrylcholinesterase; inhibitors; isatins; indanones.

\section{Introduction}

During the last decades, drugs have been developed for many human diseases. For some of them, however, there are currently only drugs available that alleviate by and large the symptoms, but they are unable to cure these diseases completely. One of those is Alzheimer's diseases (AD). In the therapy of AD, inhibitors of the enzymes acetylcholinesterase<smiles>Nc1c2c(nc3ccccc13)CCCC2</smiles>

Tacrine<smiles>CCN(C)C(=O)Oc1cccc(C(C)(C)N(C)C)c1</smiles>

Rivastigmine
(AChE) and butyrylcholinesterase (BChE) have proved particularly effective as they slow down the course of the disease, and they allow the symptoms of the disease to be alleviated over a longer period of time. Thus, at least part of the quality of life can be preserved. Among others (as depicted in Fig.1), donepezil is a well-known AChE-inhibitor ${ }^{1-3}$ that has been used for several years as medication for AD.
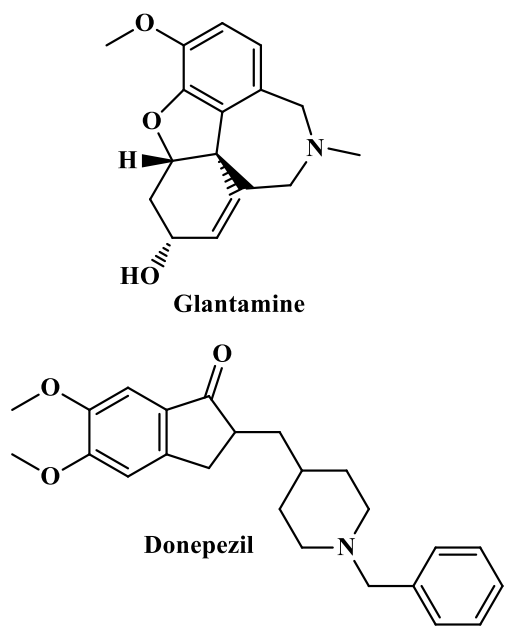

Figure 1. Today's most described drugs to alleviate AD

Despite some progress, there is still a need to find compounds being good dual inhibitors for

*Corresponding author: René Csuk

Email address: rene.csuk@chemie.uni-halle.de DOI: http://dx.doi.org/10.13171/mjc10202002161233rc acetylcholinesterase as well as for butyrylcholinesterase. In continuation of our previous 
research on enzyme inhibitors, we became interested in isatins and analogs, since for several compounds holding an indene scaffold interesting biological properties have been reported ${ }^{2,4-7}$. These compounds are also similar to donepezil, and hence it seemed reasonable to investigate their ability to inhibit $\mathrm{AChE}$ and/or BChE. As a prerequisite, however, their toxicity/cytotoxicity must be low before they can be used as a drug at a later stage.

\section{Results and iscussion}

A small library of alkyl indanone-amides was accessed from commercially available 3,4-dimethoxybenzaldehyde and $(E)$-cinnamic acid as depicted in Scheme 1.<smiles>COc1ccc(C=O)cc1OC</smiles><smiles>C#CCC#N</smiles><smiles>O=C(O)/C=C/c1ccccc1</smiles><smiles>CC(C)(C)C1(C)CCCCC1</smiles><smiles>O=C(O)CCc1ccccc1</smiles><smiles>C#CCCCC</smiles>

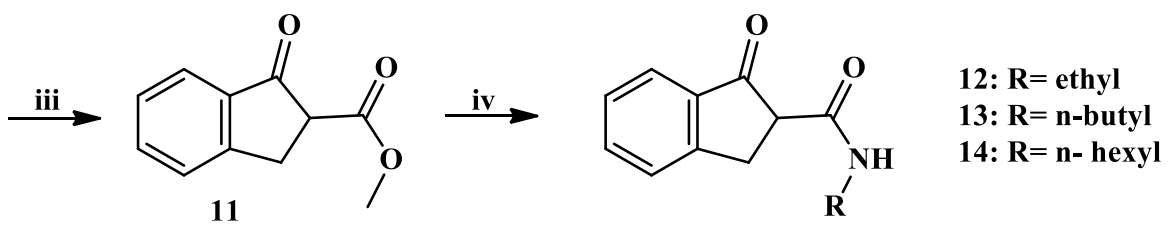<smiles>CC(C)C</smiles><smiles>[R]NC(=O)C1Cc2cc(OC)c(OC)cc2C1=O</smiles>

7: $\mathrm{R}=\mathrm{n}-$ hexyl 8: $\mathbf{R}=$ 2-benzoylphenyl

Scheme 1. Synthesis and derivatization of 5,6-dimethoxy-1-indanone: i: malonic acid, piperidine, pyridine, reflux $120^{\circ} \mathrm{C}, 4 \mathrm{~h}, 80 \%$; ii: $\mathrm{Pd} / \mathrm{C}(10 \%), \mathrm{H}_{2}$, THF, rt, overnight, 82-90\%; iii: 1) oxalylchloride, $\mathrm{CH}_{2} \mathrm{Cl}_{2}, \mathrm{rt}_{\text {, }}$ overnight 2) $\mathrm{AlCl}_{3}, \mathrm{CH}_{2} \mathrm{Cl}_{2}, 0^{\circ} \mathrm{C}, 24-53 \%$; iv: dimethylcarbonate, $\mathrm{NaH}$, reflux $90^{\circ} \mathrm{C}, 32-78 \%$; v: appropriate amine, 1,4-dioxane, microwave, 8-90\%

Thereby, 1 was obtained from a Knoevenagelcondensation in good yields of $80 \%$. Hydrogenation of $\mathbf{1}$ and $(E)$-cinnamic acid gave compounds $\mathbf{2}$ and $\mathbf{9}$ in excellent yields (82\% and $90 \%)$, respectively. Following the procedure, as described by Koca et al. ${ }^{4}$ compounds $\mathbf{4}$ and $\mathbf{1 1}$ were used as starting materials for the straightforward synthesis of several amides. We paid particular attention to the synthesis of alkyl amides, as their biological properties are mainly unexplored. Yields in these reactions depended strongly on the length of the alkyl moiety as well as on the substitution pattern of the indanone.

In the second series of compounds, substituted isatins and their oximes were synthesized starting from suitably substituted anilines using Sandmeyer reaction conditions (Scheme 2). Thus, isatins 15a-15r were obtained in yields ranging between 9 and $94 \%{ }^{8}$. Compounds 16a-16h and 17a-17d were prepared similarly; the starting materials (brominated at C-5 or $\mathrm{C}-5$ and $\mathrm{C}-7)$ were obtained adapting the procedure reported ${ }^{9}$ by Tingare et al.

The oximes were synthesized following the procedure outlined by Campbell and Warawa ${ }^{10}$. Thus, compounds 18a-18n could be synthesized in 45-94\% isolated yield while their synthesis using other procedures ${ }^{11-13}$ resulted in significantly lower yields. 
<smiles>[R4]c1c([R4])c([R4])c2c(c1[R4])NC(=O)C2=O</smiles><smiles>[R3]c1c([R4])c2c(c([R])c1Br)C(=O)C(=O)N2</smiles>

16 a-h<smiles>[R3]c1c(Br)c([R1])c2c(c1Br)NC(=O)C2=O</smiles>

17 a-d

Scheme 2. Synthetic pathway from anilines to oximes via isatin derivatives: i: 1) chloralhydrate, hydroxylamine hydrochloride, $\mathrm{Na}_{2} \mathrm{SO}_{4}$, rt; 2) $\mathrm{H}_{2} \mathrm{SO}_{4}, \mathrm{H}_{2} \mathrm{O}, 0^{\circ} \mathrm{C}$, 9-94\%; ii: $\mathrm{Br}_{2}, \mathrm{AcOH}(1 \mathrm{M}), 0^{\circ} \mathrm{C}, 3-96 \%$; iii: hydroxylamine hydrochloride, ethanol, $80^{\circ} \mathrm{C}, 45-94 \%$

Table 1. Substitution patterns for isatins (15), 5-Br-isatins (16), 5,7-Br-isatins (17) and oximes (18) as depicted in Scheme 2.

\begin{tabular}{|c|c|c|c|c|}
\hline & $\mathrm{R}^{1}$ & $\mathrm{R}^{2}$ & $\mathrm{R}^{3}$ & $\mathrm{R}^{4}$ \\
\hline $\mathrm{a}$ & $\mathrm{Cl}$ & $\mathrm{H}$ & $\mathrm{H}$ \\
\hline $\mathrm{b}$ & $\mathrm{H}$ & $\mathrm{H}$ & $\mathrm{Cl}$ \\
\hline $\mathrm{c}$ & $\mathrm{I}$ & $\mathrm{H}$ & $\mathrm{H}$ & $\mathrm{H}$ \\
\hline $\mathrm{d}$ & $\mathrm{H}$ & $\mathrm{H}$ & $\mathrm{I}$ & $\mathrm{I}$ \\
\hline $\mathrm{e}$ & $\mathrm{H}$ & $\mathrm{H}$ & $\mathrm{H}$ \\
\hline $\mathrm{f}$ & $\mathrm{H}$ & $\mathrm{H}$ & $\mathrm{F}$ \\
\hline $\mathrm{g}$ & $\mathrm{H}$ & $\mathrm{F}$ & $\mathrm{F}$ \\
\hline $\mathrm{i}$ & $\mathrm{H}$ & $\mathrm{H}$ & $\mathrm{H}$ \\
\hline $\mathrm{j}$ & $\mathrm{H}$ & $\mathrm{F}$ & $\mathrm{H}$ \\
\hline $\mathrm{k}$ & $\mathrm{He}$ & $\mathrm{H}$ & $\mathrm{H}$ \\
\hline $\mathrm{l}$ & $\mathrm{H}$ & $\mathrm{H}$ & $\mathrm{Me}$ & $\mathrm{H}$ \\
\hline $\mathrm{m}$ & $\mathrm{Br}$ & $\mathrm{He}$ & $\mathrm{Br}$ \\
\hline $\mathrm{o}$ & $\mathrm{H}$ & $\mathrm{Me}$ & $\mathrm{Hr}$ \\
\hline $\mathrm{p}$ & $\mathrm{H}$ & $\mathrm{H}$ & $\mathrm{H}$ & $\mathrm{H}$ \\
\hline $\mathrm{q}$ & $\mathrm{H}$ & $\mathrm{H}$ & $\mathrm{H}$ & $\mathrm{H}$ \\
\hline
\end{tabular}

All compounds were subjected to a biological evaluation using Ellman's assays employing $\mathrm{AChE}$ (from electrophorus electricus) and $\mathrm{BChE}$ (from equine serum); galantamine hydrobromide $(\mathrm{GH})$ was used as a standard. The results from these assays are summarized in Table 1 . These assays were performed as previously described ${ }^{14,15}$. 
Table 2. Inhibition of AChE (from electrophorus electricus) and BChE (from equine serum) as determined in Ellman's assays; inhibition constants $\mathrm{K}_{\mathrm{i}}$ and $\mathrm{K}_{\mathrm{i}^{\prime}}$ are reported in $\mu \mathrm{M}$, and $\mathbf{G H}$ (galantamine hydrobromide) was used as a standard; the results are mean values resulting from triplicate experiments; \% inhibition was determined at concentration of $50 \mu \mathrm{M}$.

\begin{tabular}{|c|c|c|c|c|c|c|}
\hline & \multicolumn{3}{|c|}{ AChE } & \multicolumn{3}{|c|}{ BChE } \\
\hline & $\mathrm{K}_{\mathrm{i}}[\mu \mathrm{M}]$ & $\mathrm{K}_{\mathrm{i}^{\prime}}[\mu \mathrm{M}]$ & \multirow{2}{*}{$\begin{array}{l}\text { type of } \\
\text { inhibition }\end{array}$} & $\mathrm{K}_{\mathrm{i}}[\mu \mathrm{M}]$ & $\mathrm{K}_{\mathrm{i}^{\prime}}[\mu \mathrm{M}]$ & \multirow{2}{*}{$\begin{array}{c}\text { type of } \\
\text { inhibition }\end{array}$} \\
\hline & \multicolumn{2}{|c|}{ (\% inhibition) } & & \multicolumn{2}{|c|}{ (\% inhibition) } & \\
\hline $\mathrm{GH}$ & $0.54 \pm 0.01$ & & competitive & $9.37 \pm 0.67$ & & competitive \\
\hline 1 & $(43.7)$ & & & $(12.3)$ & & \\
\hline 2 & $(7.22)$ & & & $(1.9)$ & & \\
\hline 3 & $7.19 \pm 0.33$ & $>100(37.7)$ & mixed & $(9.2)$ & & \\
\hline 4 & $18.95 \pm 2.19$ & $55.72 \pm 3.57$ & mixed & (13.1) & & \\
\hline 5 & $12.62 \pm 1.25$ & $121.64 \pm 8.91$ & mixed & $(10.4)$ & & \\
\hline 6 & $17.01 \pm 1.73$ & $>100(33.2)$ & mixed & (12.4) & & \\
\hline 7 & $19.42 \pm 2.35$ & & competitive & (11.9) & & \\
\hline 8 & $20.62 \pm 0.57$ & $62.23 \pm 2.44$ & mixed & (14.7) & & \\
\hline 9 & $(7.4)$ & & & $(2.2)$ & & \\
\hline 10 & $(10.08)$ & & & $(3.3)$ & & \\
\hline 11 & $(9.43)$ & & & $(4.0)$ & & \\
\hline 12 & $16.70 \pm 2.39$ & $>70$ & mixed & $(1.8)$ & & \\
\hline 13 & $18.52 \pm 1.18$ & $>70$ & mixed & $(7.5)$ & & \\
\hline 14 & $12.33 \pm 1.32$ & $>70$ & mixed & $(5.6)$ & & \\
\hline
\end{tabular}

The results from the Ellman's assays for the isatin derivatives are summarized in Table 3, and only moderate activity was determined for several of the compounds. However, the results for compound 17c were outstanding, since this compound was shown to be an excellent mixed-type inhibitor for AChE. As far as $\mathrm{BChE}$ is concerned, only a small number of substances $(\mathbf{1 7 a}, \mathbf{1 7} \mathbf{c}$, some of $\mathbf{1 8})$ were found to be a good or at least moderate inhibitor for this enzyme. Furthermore, only a few compounds of this series hold the rare property to bind to both enzymes.

Remarkable is the difference between compounds $\mathbf{1 7 a}$ and $17 \mathbf{c}$ in their ability to inhibit AChE. To get an explanation, we performed additional molecular modelling calculations using AutoDock. These calculations showed for both compounds a high affinity to the enzyme; however, the affinity of 17c (holding an iodine substituent) to the active site is higher than that of 17a (with a chlorine substituent); also, the tendency of $\mathbf{1 7 a}$ to get stuck in its access to the active site seems higher than that of 17c. The results of these calculations are shown in Fig.2. Binding scores for the different conformations of $\mathbf{1 7 a}$ and $17 \mathbf{c}$ were calculated, and for $17 \mathbf{a}-7.62$ to $-7.89 \mathrm{kcal} / \mathrm{mol}$ and for $17 \mathrm{c}-7.38$ to $-7.40 \mathrm{kcal} / \mathrm{mol}$ were found.

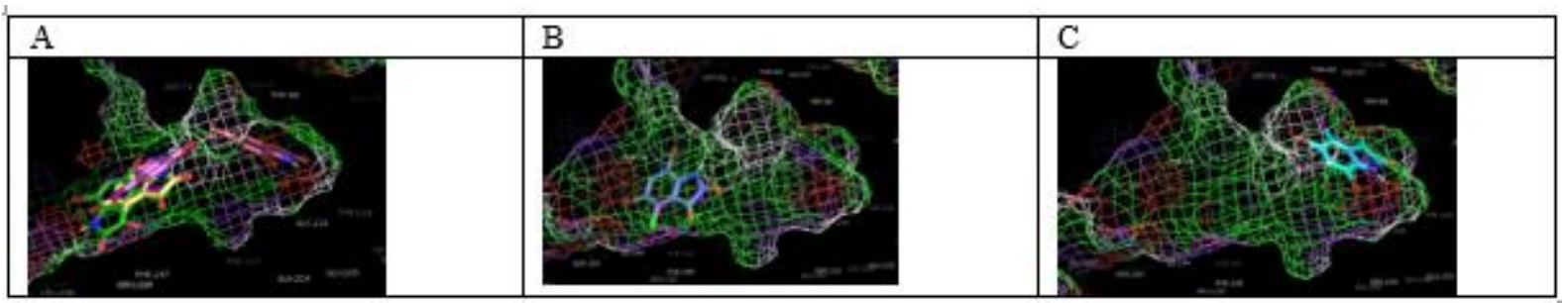

Figure 2. Depiction of the results from the molecular modelling calculations. A: yellow: compound 17a; brown, green and pink: 3 possible conformations of 17c; B: location of $18 \mathrm{a}$ "being stuck" in the access channel to the active site; $\mathrm{C}$ : Location of $\mathbf{1 7} \mathbf{c}$ in the active site

Table 3. AChE (from electrophorus electricus)- and BChE (from equine serum)- inhibition determined in Ellman's assay; $\mathrm{K}_{\mathrm{i}}$ and $\mathrm{K}_{\mathrm{i}^{\prime}}$ (inhibition constants) in $\mu \mathrm{M}$ for $\mathbf{G H}$ (galantamine hydrobromide as a standard) and compounds 15-18; averaged from triplicate experiments; \% inhibition were determined at a concentration of $50 \mu \mathrm{M}$.

\begin{tabular}{|c|c|c|c|c|c|c|}
\hline & \multicolumn{3}{|c|}{ AChE } & \multicolumn{3}{c|}{ BChE } \\
\hline & $\mathrm{K}_{\mathrm{i}}[\mu \mathrm{M}]$ & $\mathrm{K}_{\mathrm{i}^{\prime}}[\mu \mathrm{M}]$ & $\begin{array}{c}\text { type of } \\
\text { inhibition }\end{array}$ & $\mathrm{K}_{\mathrm{i}}[\mu \mathrm{M}]$ & $\mathrm{K}_{\mathrm{i}^{\prime}}[\mu \mathrm{M}]$ & $\begin{array}{c}\text { type of } \\
\text { inhibition }\end{array}$ \\
\hline $\mathrm{GH}$ & $0.54 \pm 0.01$ & & competitive & $9.37 \pm 0.67$ & & competitive \\
\hline
\end{tabular}




\begin{tabular}{|c|c|c|c|c|c|c|}
\hline $15 \mathrm{a}$ & $13.49 \pm 2.27$ & $27.11 \pm 8.01$ & mixed & $20.14 \pm 1.04$ & & competitive \\
\hline $15 b$ & $29.72 \pm 8.90$ & $127.01 \pm 3.74$ & mixed & $32.76 \pm 4.40$ & & competitive \\
\hline $15 c$ & $17.72 \pm 4.06$ & $145.04 \pm 9.13$ & mixed & $>100$ & & \\
\hline $15 d$ & $21.41 \pm 1.82$ & & competitive & $>100$ & & \\
\hline $15 \mathrm{e}$ & $15.29 \pm 0.14$ & & competitive & $>100$ & & \\
\hline $15 f$ & $13.24 \pm 0.78$ & $64.29 \pm 3.25$ & mixed & $42.52 \pm 2.67$ & $228.85 \pm 16.60$ & mixed \\
\hline $15 \mathrm{~g}$ & $>100$ & & & $>100$ & & \\
\hline $15 \mathrm{~h}$ & $>100$ & & & $>100$ & & \\
\hline $15 \mathrm{i}$ & $20.04 \pm 1.15$ & $111.88 \pm 4.46$ & mixed & $>100$ & & \\
\hline $15 \mathrm{j}$ & $24.47 \pm 5.55$ & $106.71 \pm 7.62$ & mixed & $>100$ & & \\
\hline $15 \mathrm{k}$ & $18.94 \pm 1.59$ & $85.68 \pm 3.68$ & mixed & $>100$ & & \\
\hline 151 & $24.12 \pm 3.18$ & & competitive & $>100$ & & \\
\hline $15 \mathrm{~m}$ & $18.45 \pm 2.53$ & $123.80 \pm 29.28$ & mixed & $>100$ & & \\
\hline $15 n$ & $17.03 \pm 3.28$ & $186.40 \pm 18.10$ & mixed & $>100$ & & \\
\hline 160 & $>100$ & & & $>100$ & & \\
\hline $15 \mathrm{p}$ & $>100$ & & & $17.09 \pm 3.46$ & & competitive \\
\hline $15 q$ & $>100$ & & & $>100$ & & \\
\hline $15 r$ & $24.56 \pm 5.74$ & $108.67 \pm 7.13$ & mixed & $>100$ & & \\
\hline $16 a$ & (14.9) & & & $(17.7)$ & & \\
\hline $16 b$ & $(48.9)$ & & & $(8.6)$ & & \\
\hline $16 \mathrm{c}$ & $(17.2)$ & & & $(23.7)$ & & \\
\hline $16 \mathrm{~d}$ & $13.14 \pm 2.31$ & $12.51 \pm 0.90$ & mixed & $>60$ & & \\
\hline $16 \mathrm{e}$ & (15.9) & & & $(8.6)$ & & \\
\hline $16 f$ & (34.2) & & & $(17.0)$ & & \\
\hline $16 \mathrm{~g}$ & $(15.2)$ & & & (1.4) & & \\
\hline $17 \mathrm{a}$ & $10.54 \pm 0.76$ & $34.42 \pm 1.25$ & mixed & $5.38 \pm 0.88$ & $44.35 \pm 7.28$ & mixed \\
\hline \multirow[t]{2}{*}{$17 \mathrm{c}$} & $2.52 \pm 0.61$ & $11.74 \pm 1.31$ & mixed & $4.49 \pm 0.32$ & $6.56 \pm 0.57$ & mixed \\
\hline & $>100$ & & & $98.93 \pm 19.93$ & $135.37 \pm 12.80$ & mixed \\
\hline $18 \mathrm{~b}$ & $>100$ & & & $16.65 \pm 1.38$ & & competitive \\
\hline $18 \mathrm{c}$ & $>100$ & & & $65.60 \pm 16.80$ & $68.62 \pm 11.05$ & mixed \\
\hline $18 \mathrm{~d}$ & $>80(17.6)$ & & & $14.50 \pm 0.48$ & $106.02 \pm 6.93$ & mixed \\
\hline $18 \mathrm{e}-\mathrm{i}$ & $>100$ & & & $>100$ & & \\
\hline $18 \mathrm{j}$ & $29.70 \pm 2.68$ & & competitive & $>100$ & & \\
\hline $18 \mathrm{k}$ & $>100$ & & & $>100$ & & \\
\hline 181 & $>100$ & & & $>100$ & & \\
\hline $18 \mathrm{~m}$ & $>100$ & & & $22.58 \pm 8.34$ & $76.11 \pm 15.55$ & mixed \\
\hline $19 n$ & $>100$ & & & $>100(16.9)$ & & \\
\hline
\end{tabular}

Table 4. Cytotoxicity of indanone compounds 1-14; $\mathrm{SRB}$ assay $\mathrm{EC}_{50}$ values $[\mu \mathrm{M}]$ after $96 \mathrm{~h}$ of treatment; averaged from three independent experiments performed each in triplicate; confidence interval $\mathrm{CI}=95 \%$. Human cancer cell lines: FaDu (hypopharyngeal carcinoma), A2780 (ovarian carcinoma), HT29 (colorectal carcinoma), MCF-7 (breast carcinoma), SW1736 (thyroid carcinoma), A375 (malignant melanoma) and non-malignant mouse fibroblasts (NiH 3T3); cut-off $30 \mu \mathrm{M}$.

\begin{tabular}{|c|c|c|c|c|c|c|c|}
\hline & FaDu & A2780 & HT29 & MCF-7 & SW1736 & A375 & NIH 3T3 \\
\hline $1-11$ & $>30$ & $>30$ & $>30$ & $>30$ & $>30$ & $>30$ & $>30$ \\
\hline 12 & $>30$ & $>30$ & $>30$ & $25.62 \pm 3.0$ & $>30$ & $>30$ & $>30$ \\
\hline 13 & $>30$ & $>30$ & $>30$ & $23.78 \pm 1.6$ & $>30$ & $>30$ & $>30$ \\
\hline 14 & $>30$ & $>30$ & $>30$ & $4.28 \pm 0.5$ & $>30$ & $>30$ & $>30$ \\
\hline
\end{tabular}

In vitro cytotoxicity of the indanones 1-14 and isatinderivatives 15-18 was screened in colorimetric SRB- assays, and the $\mathrm{EC}_{50}$ values were determined using several human tumor cell lines and non-malignant 
mouse fibroblasts (NIH 3T3). The results from these assays are compiled in Tables 4 and 5

No cytotoxicity was observed for compounds 1-11 (cut-off: $30 \mu \mathrm{M}$ ), and compounds $\mathbf{1 2}$ and $\mathbf{1 3}$ showed moderate activity. Compound 14, however, while being not cytotoxic for several human tumor cell lines, exhibited selective and good cytotoxicity for the human adenocarcinoma breast cell lines MCF-7.

Table 5. Cytotoxicity of isatin compounds 15-18 SRB assay $\mathrm{EC}_{50}$ values $[\mu \mathrm{M}]$ after $96 \mathrm{~h}$ of treatment; averaged from three independent experiments performed each in triplicate; confidence interval $\mathrm{CI}=95 \%$. Human cancer cell lines: FaDu (hypopharyngeal carcinoma), A2780 (ovarian carcinoma), HT29 (colorectal carcinoma), MCF7 (breast carcinoma), SW1736 (thyroid carcinoma), 8505C (thyroid anaplastic carcinoma), and non-malignant mouse fibroblasts (NIH 3T3); cut-off $30 \mu \mathrm{M}$.

\begin{tabular}{|c|c|c|c|c|c|c|c|c|}
\hline & FaDu & $518 \mathrm{~A} 2$ & $\mathrm{~A} 2780$ & HT29 & MCF7 & A549 & $8505 \mathrm{C}$ & NiH3T3 \\
\hline $15 \mathrm{a}-\mathrm{c}$ & - & $>30$ & $>30$ & $>30$ & $>30$ & $>30$ & $>30$ & $>30$ \\
\hline $15 \mathrm{~d}$ & - & $9.2 \pm 0.7$ & $10.6 \pm 0.3$ & $24.8 \pm 1.1$ & $17.4 \pm 1.7$ & $>30$ & $>30$ & $>30$ \\
\hline $15 \mathrm{e}$ & - & 23.7 & $>30$ & $>30$ & $>30$ & $>30$ & $>30$ & $>30$ \\
\hline $15 \mathrm{f}$ & - & $16.7 \pm 0.6$ & $25.5 \pm 1.2$ & $>30$ & $>30$ & $>30$ & $>30$ & $>30$ \\
\hline $15 \mathrm{~g}, \mathrm{~h}$ & - & $>30$ & $>30$ & $>30$ & $>30$ & $>30$ & $>30$ & $>30$ \\
\hline $15 \mathrm{i}$ & - & $14.9 \pm 0.4$ & $12.1 \pm 0.7$ & $23.8 \pm 1.7$ & $21.5 \pm 3.0$ & $18.2 \pm 1.1$ & $27.4 \pm 0.4$ & $15.2 \pm 1.6$ \\
\hline $15 \mathrm{j}-1$ & - & $>30$ & $>30$ & $>30$ & $>30$ & $>30$ & $>30$ & $>30$ \\
\hline $15 \mathrm{~m}$ & - & $8.6 \pm 0.8$ & $9.1 \pm 0.4$ & $22.6 \pm 2.5$ & $19.7 \pm 2.1$ & $>30$ & $>30$ & $>30$ \\
\hline $15 \mathrm{n}$ & - & $27.3 \pm 0.6$ & $>30$ & $>30$ & $>30$ & $>30$ & $>30$ & $>30$ \\
\hline $15 \mathrm{o}$ & - & $9.8 \pm 0.6$ & $>30$ & $>30$ & $>30$ & $24.4 \pm 1.6$ & $12.0 \pm 0.9$ & $>30$ \\
\hline $15 \mathrm{p}$ & - & $6.5 \pm 0.6$ & $7.3 \pm 0.6$ & $30.3 \pm 2.4$ & $25.5 \pm 2.9$ & $>30$ & $>30$ & $28.7 \pm 3.0$ \\
\hline $15 \mathrm{q}, \mathrm{r}$ & $>30$ & $>30$ & $>30$ & $>30$ & $>30$ & $>30$ & $>30$ & $>30$ \\
\hline $16 \mathrm{a}$ & $>30$ & $17.2 \pm 0.2$ & $>30$ & $>30$ & $25.7 \pm 0.2$ & $13.9 \pm 1.3$ & $16.6 \pm 0.1$ & $19.9 \pm 0.9$ \\
\hline $16 \mathrm{~b}$ & $10.9 \pm 2.1$ & - & $7.7 \pm 1.8$ & - & $13.7 \pm 0.9$ & - & - & - \\
\hline $16 \mathrm{c}$ & $14.6 \pm 1.4$ & - & $9.9 \pm 0.6$ & - & $16.1 \pm 0.8$ & - & - & - \\
\hline $16 \mathrm{~d}$ & $19.6 \pm 2.8$ & - & $13.3 \pm 2.1$ & - & $20.5 \pm 1.1$ & - & - & - \\
\hline $16 \mathrm{e}$ & - & $10.6 \pm 1.9$ & $>30$ & $>30$ & $>30$ & $>30$ & $17.7 \pm 0.6$ & $>30$ \\
\hline $16 \mathrm{f}$ & - & $9.0 \pm 0.6$ & $>30$ & $>30$ & $>30$ & $22.0 \pm 0.8$ & $12.8 \pm 0.2$ & $>30$ \\
\hline $16 \mathrm{~g}$ & $8.95 \pm 0.7$ & - & $6.47 \pm 0.8$ & - & $12.29 \pm 1.0$ & - & - & - \\
\hline & $\mathbf{F a D u}$ & $\mathbf{5 1 8 A 2}$ & $\mathbf{A 2 7 8 0}$ & $\mathbf{H T 2 9}$ & $\mathbf{M C F} 7$ & $\mathbf{A 5 4 9}$ & $\mathbf{N i H 3 T 3}$ \\
\hline $17 \mathrm{a}-\mathrm{d}$ & $>30$ & $>30$ & $>30$ & $>30$ & $>30$ & $>30$ & $>30$ & $>$ \\
\hline $18 \mathrm{a}-\mathrm{n}$ & $>30$ & $>30$ & $>30$ & $>30$ & $>30$ & $>30$ & $>30$ & $>$ \\
\hline
\end{tabular}

For several of the isatins $\mathbf{1 5 - 1 8}$ only low or no cytotoxicity was found. This is a prerequisite for the possible use of these compounds as inhibitors of cholinesterases in ongoing investigations presently studied in more detail in our laboratories.

\section{Conclusion}

A small library consisting of 49 indanone-amides and substituted isatin derivatives has been investigated for the ability of these compounds to act as inhibitors for the enzymes acetyl- and butyrylcholinesterase (AChE, BChE). Among these compounds, 5,7-dibromo-4-iodoisatin (17c) was shown to be a good mixed-type inhibitor for AChE $\left(\mathrm{K}_{\mathrm{i}}=2.52 \pm 0.61 \mu \mathrm{M}\right.$ and $\left.\mathrm{K}_{\mathrm{i}^{\prime}}=11.74 \pm 1.31 \mu \mathrm{M}\right)$ but this compound also acted as a dual inhibitor for $\mathrm{BChE}$ $\left(\mathrm{K}_{\mathrm{i}}=4.49 \pm 0.32 \mu \mathrm{M}\right.$ and $\left.\mathrm{K}_{\mathrm{i}^{\prime}}=6.56 \pm 0.57 \mu \mathrm{M}\right)$. Interestingly, $N$-hexyl-1-oxo-2,3-dihydro-1H-indene2-carboxamide (14) was cytotoxic especially for MCF-7 breast adenocarcinoma cells $\left(\mathrm{EC}_{50}=4.28 \mu \mathrm{M}\right)$.

\section{Experimental}

NMR spectra were recorded using the Varian spectrometers Gemini 2000 or Unity 500 ( $\delta$ given in ppm, $J$ in $\mathrm{Hz}$; typical experiments: $\mathrm{H}-\mathrm{H}-\mathrm{COSY}$, HMBC, HSQC, NOESY), MS spectra were taken on a Finnigan MAT LCQ 7000 (electrospray, voltage $4.1 \mathrm{kV}$, sheath gas nitrogen) instrument. TLC was performed on silica gel (Merck 5554, detection with cerium molybdate reagent); melting points are uncorrected (Leica hot stage microscope or BÜCHI Melting Point M-565), and elemental analyses were performed on a Foss-Heraeus Vario EL (CHNS) unit. IR spectra were recorded on a Perkin Elmer FT-IR spectrometer Spectrum 1000 or on a Perkin-Elmer Spectrum Two (UATR Two Unit). The solvents were dried according to usual procedures. The purity of the compounds was determined by HPLC and found to be $>96 \%$. 
(2E)-3-(3,4-Dimethoxyphenyl)-2-propenoic acid (1)

To a solution of 3,4-dimethoxybenzaldehyde $(8.30 \mathrm{~g}, 50 \mathrm{mmol})$ and malonic acid $(6.25 \mathrm{~g}, 60 \mathrm{mmol})$ in pyridine $(125 \mathrm{~mL})$ at $120^{\circ} \mathrm{C}$ piperidine $(0.2 \mathrm{~mL}, 2 \mathrm{mmol})$ was added, and the reaction mixture was heated under reflux for $4 \mathrm{~h}$. Usual aqueous workup followed by re-crystallization from EtOH gave $\mathbf{1}^{16}$ $(8.28 \mathrm{~g}, 80 \%)$ as an off-white solid;

$\mathrm{R}_{F}=0.05$ (silica gel, $n$-hexane/ethyl acetate, 4:1); $\mathrm{mp}=178-180^{\circ} \mathrm{C}$ (lit.: $\left.{ }^{17} 181-183^{\circ} \mathrm{C}\right)$;

MS (ESI, MeOH): m/z $(\%)=207.0\left([\mathrm{M}-\mathrm{H}]^{-}, 11\right)$, 415.1 ([2M-H] $\left.]^{-}, 100\right), 436.9$ ([2M-2H+Na] $\left.]^{-}, 13\right)$.

\section{3-(3,4-Dimethoxyphenyl)propanoic acid (2)}

A solution of 1 (1 g, $4.8 \mathrm{mmol})$ in dry THF $(20 \mathrm{~mL})$ was hydrogenated overnight at $75 \mathrm{psi}$ in the presence of $\mathrm{Pd} / \mathrm{C}(10 \%, 0.116 \mathrm{~g})$. Usual work-up gave $\mathbf{2}$ $(0.83 \mathrm{~g}, 82 \%)^{18}$ as a white solid;

$\mathrm{R}_{F}=0.09$ (silica gel, $n$-hexane/ethyl acetate, $4: 1$ ); $\mathrm{mp}=98-99^{\circ} \mathrm{C}$ (lit.: $\left.{ }^{19} 98-99^{\circ} \mathrm{C}\right)$;

MS (ESI, MeOH): m/z $(\%)=209.1\left([\mathrm{M}-\mathrm{H}]^{-}, 100\right)$, $441\left([2 \mathrm{M}-2 \mathrm{H}+\mathrm{Na}]^{-}, 71\right)$.

\section{5,6-Dimethoxy-indane-1-one (3)}

To an ice-cold solution of $2(0.5 \mathrm{~g}, 2.37 \mathrm{mmol})$ in dry dichloromethane $(8 \mathrm{~mL})$, oxalyl chloride $(0.803 \mathrm{~mL}$, $9.5 \mathrm{mmol}$ ) and dimethylformamide (5 drops) were added. After an additional stirring at room temperature for $1 \mathrm{~h}$, the solvents were evaporated. The residue was dissolved in dry dichloromethane $(8 \mathrm{~mL})$, and at $0^{\circ} \mathrm{C} \mathrm{AlCl}_{3}(0.57 \mathrm{~g}, 4.3 \mathrm{mmol})$ was added in several portions. After stirring at room temperature for $1 \mathrm{~h}$ at room temperature followed by usual workup and re-crystallization from $\mathrm{EtOH}$, $3(0.11 \mathrm{~g}, 24 \%){ }^{4}$ was obtained as an off-white solid; $\mathrm{R}_{F}=0.39$ (silica gel, $n$-hexane/ethyl acetate, $1: 1$ ); $\mathrm{mp}=117-118^{\circ} \mathrm{C}$ (lit.: ${ }^{20} 117-119^{\circ} \mathrm{C}$ );

MS (ESI, MeOH): m/z $(\%)=193.2\left([\mathrm{M}+\mathrm{H}]^{+}, 100\right)$, $215.0\left([\mathrm{M}+\mathrm{Na}]^{+}, 34\right)$.

\section{5,6-Dimethoxy-1-oxo-indan-2-carboxylic acid methyl ester (4)}

To a solution of $\mathbf{3}(4.00 \mathrm{~g}, 20 \mathrm{mmol})$ in dimethylcarbonate $(22 \mathrm{~mL}), \mathrm{NaH}(2.4 \mathrm{~g}, 60 \mathrm{mmol}$, $60 \%$ in mineral oil) was added, and the mixture was stirred at $90^{\circ} \mathrm{C}$ for $2 \mathrm{~h}$. Usual aqueous work-up followed by re-crystallization from $\mathrm{EtOH}$ gave 4 $(3.92 \mathrm{~g}, 78 \%)^{4}$ as lightly yellowish needles;

$\mathrm{R}_{F}=0.12$ (silica gel, chloroform); $\mathrm{mp}=160-163^{\circ} \mathrm{C}$ (lit.: ${ }^{21} 161-162^{\circ} \mathrm{C}$ );

MS (ESI, MeOH): m/z $(\%)=251.1\left([\mathrm{M}+\mathrm{H}]^{+}, 100\right)$, $273.1\left([\mathrm{M}+\mathrm{Na}]^{+}, 43\right), 277.7\left([2 \mathrm{M}+\mathrm{Na}+\mathrm{MeOH}]^{+}, 10\right)$, $285.7\left([2 \mathrm{M}+\mathrm{K}+\mathrm{H}+\mathrm{MeOH}]^{2+}, 11\right)$.

\section{General Procedure A}

To a solution of compound $4(1.2 \mathrm{mmol})$ was dissolved in dry 1,4-dioxane $(3 \mathrm{~mL})$, the corresponding amine $(1.2 \mathrm{mmol})$ was added, and the solution was sonicated in an ultrasound bath for $1 \mathrm{~min}$ followed by microwave irradiation for $10 \mathrm{~min}$ $\left(1200 \mathrm{rpm}, 300 \mathrm{~W}, 170^{\circ} \mathrm{C}\right){ }^{4}$. The solvent was removed under reduced pressure, and the residue was subjected to recrystallization from $\mathrm{EtOH}$.
Compounds 5-8 and 12-15 were prepared following procedure A.

\section{$\mathrm{N}$-Ethyl-5,6-dimethoxy-1-oxo-2,3-dihydro-1H-} indene-2-carboxamide (5)

Compound $5(0.22 \mathrm{~g}, 70 \%)$ was obtained as a white solid;

$\mathrm{R}_{F}=0.21$ (silica gel, $n$-hexane/ethyl acetate, $1: 1$ ); $\mathrm{mp}=188-192^{\circ} \mathrm{C}$,

IR (KBr): $v=3284 m, 3083 w, 2938 w, 1702 s, 1634 s$, $1592 m, 1554 m, 1503 s, 1458 m, 1363 w, 1311 s, 1267 s$, $1222 \mathrm{~m}, 1200 w, 1151 w, 1115 \mathrm{~m}, 1032 \mathrm{~m} \mathrm{~cm}^{-1}$;

UV-vis $\left(\mathrm{CHCl}_{3}\right): \lambda(\log \varepsilon)=251$ (3.57), 295 (3.96), 348 (4.06) nm;

${ }^{1} \mathrm{H}$ NMR $\left(400 \mathrm{MHz}, \mathrm{CDCl}_{3}\right): \delta=7.12(s, 1 \mathrm{H}, 7-\mathrm{H})$, $6.90(s, 1 \mathrm{H}, 4-\mathrm{H}), 3.96(s, 3 \mathrm{H}, 9-\mathrm{H}), 3.88(s, 3 \mathrm{H}, 10-$ $\mathrm{H}), 3.64(d d, J=3.6,0.9 \mathrm{~Hz}, 1 \mathrm{H}, 3 \mathrm{a}-\mathrm{H}), 3.50(d d$, $J=7.9,3.6 \mathrm{~Hz}, 1 \mathrm{H}, 2-\mathrm{H}), 3.37-3.27\left(m, 2 \mathrm{H}, 1^{\prime}-\mathrm{H}\right)$, $3.23(d d, J=17.5,7.9 \mathrm{~Hz}, 1 \mathrm{H}, 3 \mathrm{~b}-\mathrm{H}), 1.16(t, J=7.3$ $\left.\mathrm{Hz}, 3 \mathrm{H}, 2^{\prime}-\mathrm{H}\right) \mathrm{ppm} ;{ }^{13} \mathrm{C} \mathrm{NMR}\left(100 \mathrm{MHz} ; \mathrm{CDCl}_{3}\right)$ : $\delta=202.0(\mathrm{C}-1), 166.8$ (C-8), 156.6 (C-5), 150.3 (C-7a), 149.8 (C-6), 128.1 (C-3a), 107.6 (C-4), 104.6 (C-7), 56.5 (C-9), 56.2 (C-10), 53.2 (C-2), 34.8 (C-1'), 28.6 (C-3), 14.9 (C-2') ppm;

MS $(\mathrm{ESI}, \mathrm{MeOH}): \mathrm{m} / \mathrm{z}(\%)=264.1\left([\mathrm{M}+\mathrm{H}]^{+}, 46\right)$, $286.1\left([\mathrm{M}+\mathrm{Na}]^{+}, 38\right), 298.7\left([2 \mathrm{M}+\mathrm{Ca}+\mathrm{MeOH}]^{2+}, 16\right)$, $414.6\left([3 \mathrm{M}+\mathrm{Ca}]^{2+}, 30\right), 548.8\left([2 \mathrm{M}+\mathrm{Na}]^{+}, 100\right)$; analysis calcd for $\mathrm{C}_{14} \mathrm{H}_{17} \mathrm{NO}_{4}$ (263.29): C 63.87, H 6.51, N 5.32; found C 63.66, H 6.79, N 5.11.

\section{$\mathrm{N}$-Butyl-5,6-dimethoxy-1-oxo-2,3-dihydro-1H- indene-2-carboxamide (6)}

Compound $6(0.32 \mathrm{~g}, 90 \%)$ was obtained as a white solid;

$\mathrm{R}_{F}=0.57$ (silica gel, $n$-hexane/ethyl acetate, $1: 1$ );

$\mathrm{mp}=152-154^{\circ} \mathrm{C}$; IR (KBr): $v=3445 m, 3291 s$, $2925 m, 2870 w, 1704 s, 1638 s, 1592 m, 1554 m, 1505 s$, $1443 m, 1367 w, 1313 s, 1268 s, 1224 m, 1200 w, 1116 m$, $1030 \mathrm{~m} \mathrm{~cm}^{-1}$;

UV-vis $\left(\mathrm{CHCl}_{3}\right): \lambda(\log \varepsilon)=251$ (3.59), 295 (3.97), $347(4.03) \mathrm{nm}$;

${ }^{1} \mathrm{H}$ NMR (400 MHz, $\left.\mathrm{CDCl}_{3}\right): \delta=7.14(s, 1 \mathrm{H}, 7-\mathrm{H})$, $6.92(s, 1 \mathrm{H}, 4-\mathrm{H}), 3.98(s, 3 \mathrm{H}, 9-\mathrm{H}), 3.91(s, 3 \mathrm{H}, 10-$ $\mathrm{H}), 3.70(d d, J=17.6,3.5 \mathrm{~Hz}, 1 \mathrm{H}, 3 \mathrm{a}-\mathrm{H}), 3.52(d d, J$ $=7.9,3.6 \mathrm{~Hz}, 1 \mathrm{H}, 2-\mathrm{H}), 3.31(d d d, J=12.8,7.3,2.1$ $\left.\mathrm{Hz}, 2 \mathrm{H}, 1^{\prime}-\mathrm{H}\right), 3.28-3.22(m, 1 \mathrm{H}, 3 \mathrm{~b}-\mathrm{H}), 1.58-1.50$ $\left(\mathrm{m}, 2 \mathrm{H}, 2^{\prime}-\mathrm{H}\right), 1.44-1.31\left(\mathrm{~m}, 2 \mathrm{H}, 3^{\prime}-\mathrm{H}\right), 0.93(t, J=$ $\left.7.3 \mathrm{~Hz}, 3 \mathrm{H}, 4^{\prime}-\mathrm{H}\right) \mathrm{ppm} ;{ }^{13} \mathrm{C} \mathrm{NMR}\left(100 \mathrm{MHz} ; \mathrm{CDCl}_{3}\right)$ : $\delta=199.4(\mathrm{C}-1), 166.3(\mathrm{C}-8), 157.6$ (C-5), 150.2 (C7a), 149.5 (C-6), 128.0 (C-3a), 107.6 (C-4), 104.7 (C7), 56.5 (C-9), 56.2 (C-10), 53.2 (C-2), 39.7 (C-1'), 31.7 (C-2'), 28.7 (C-3), 20.3 (C-3'), 14.1 (C-4') ppm; MS (ESI, $\mathrm{MeOH}): \mathrm{m} / \mathrm{z}(\%)=292.1\left([\mathrm{M}+\mathrm{H}]^{+}, 41\right)$, $314.2\left([\mathrm{M}+\mathrm{Na}]^{+}, 27\right), 318.7\left([2 \mathrm{M}+\mathrm{Na}+\mathrm{H}+\mathrm{MeOH}]^{2+}\right.$, $3), \quad 326.7 \quad\left([2 \mathrm{M}+\mathrm{Ca}+\mathrm{MeOH}]^{2+}, \quad 13\right), \quad 448.6$ $\left([3 \mathrm{M}+\mathrm{Na}+\mathrm{H}]^{2+}, 6\right), 456.6\left([3 \mathrm{M}+\mathrm{Ca}]^{2+}, 29\right), 604.9$ $\left.\left([2 \mathrm{M}+\mathrm{Na}]^{+}\right), 100\right)$;

analysis calcd for $\mathrm{C}_{16} \mathrm{H}_{21} \mathrm{NO}_{4}(291.35)$ : C $65.96, \mathrm{H}$ 7.27, N 4.81; found C 65.77, H 7.45, N 4.51.

\section{$\mathrm{N}$-Hexyl-5,6-dimethoxy-1-oxo-2,3-dihydro-1H- indene-2-carboxamide (7)}

Compound $7(0.04 \mathrm{~g}, 11 \%)$ was obtained as a white solid; 
$\mathrm{R}_{F}=0.53$ (silica gel, $n$-hexane/ethyl acetate, 1:1); $\mathrm{mp}=136-139^{\circ} \mathrm{C}$;

IR (KBr): $v=3752 w, 3441 m, 3289 m, 2930 m, 1702 m$, $1636 s, 1592 m, 1504 s, 1458 m, 1312 s, 1268 s, 1223 m$, $1115 \mathrm{~m}, 1031 \mathrm{~m} \mathrm{~cm}^{-1}$; UV-vis $\left(\mathrm{CHCl}_{3}\right): \lambda(\log \varepsilon)=251$ (3.60), 294 (3.96), 346 (4.02) nm;

${ }^{1} \mathrm{H}$ NMR (400 MHz, $\left.\mathrm{CDCl}_{3}\right): \delta=7.14(s, 1 \mathrm{H}, 7-\mathrm{H})$, $6.91(s, 1 \mathrm{H}, 4-\mathrm{H}), 3.98(s, 3 \mathrm{H}, 9-\mathrm{H}), 3.90(s, 3 \mathrm{H}, 10-$ $\mathrm{H}), 3.70\left(d d, J=17.4,3.5 \mathrm{~Hz}, 1 \mathrm{H}, 3_{\mathrm{a}}-\mathrm{H}\right), 3.52(d d, J$ $=7.9,3.6 \mathrm{~Hz}, 1 \mathrm{H}, 2-\mathrm{H}), 3.35-3.23\left(m, 2 \mathrm{H}, 1^{\prime}-\mathrm{H}\right)$, $3.25(d d, J=17.4 \mathrm{~Hz}, 7.7 \mathrm{~Hz}, 1 \mathrm{H}, 3 \mathrm{~b}-\mathrm{H}), 1.54$ $\left(p, J=7.1 \mathrm{~Hz}, 2 \mathrm{H}, 2^{\prime}-\mathrm{H}\right), 1.37-1.31\left(m, 2 \mathrm{H}, 3^{\prime}-\mathrm{H}\right)$, $1.31-1.27$ (m, 4H, 4'-H + 5'-H), 0.90-0.86 (m, 3H, $\left.6^{\prime}-\mathrm{H}\right) \mathrm{ppm} ;{ }^{13} \mathrm{C} \mathrm{NMR}\left(100 \mathrm{MHz} ; \mathrm{CDCl}_{3}\right): \delta=202.1$ (C-1), 166.8 (C-8), 156.6 (C-5), 150.3 (C-7a), 149.8 (C-6), 128.2 (C-3a), 107.6 (C-4), 104.6 (C-7), 56.5 (C-9), 56.3 (C-10), 53.2 (C-2), 40.0 (C-1'), 31.6 (C4'), 29.6 (C-2'), 28.7 (C-3), 26.7 (C-3'), 22.7 (C-5'), $14.2\left(\mathrm{C}^{-} 6^{\prime}\right) \mathrm{ppm}$;

MS (ESI, $\mathrm{MeOH}): \mathrm{m} / \mathrm{z}(\%)=320.2\left([\mathrm{M}+\mathrm{H}]^{+}, 32\right)$, $342.2\left([\mathrm{M}+\mathrm{Na}]^{+}, 16\right), 354.8\left([2 \mathrm{M}+\mathrm{K}+\mathrm{H}+\mathrm{MeOH}]^{2+}\right.$, 5), $498.7\left([3 \mathrm{M}+\mathrm{Ca}]^{2+}, 13\right), 660.9\left([2 \mathrm{M}+\mathrm{Na}]^{+}, 100\right)$; analysis calcd for $\mathrm{C}_{18} \mathrm{H}_{25} \mathrm{NO}_{4}$ (319.40): C 67.69, H 7.89, N 4.39; found C 67.50, H 8.02, N 4.11.

\section{N-(2-Benzoylphenyl)-5,6-dimethoxy-1-oxo-2,3- dihydro-1H-indene-2-carboxamide $(8)$}

Compound $8(0.12 \mathrm{~g}, 70 \%)$ was obtained as a white solid;

$\mathrm{R}_{F}=0.5$ (silica gel, $n$-hexane/ethyl acetate, $1: 1$ ); $\mathrm{mp}=190-192^{\circ} \mathrm{C}$;

IR (KBr): $v=3517 m, 3228 m, 2923 w, 2850 w, 1686 s$, $1590 m, 1502 m, 1450 w, 1371 w, 1315 s, 1276 m$, $1224 w, 1191 w, 1114 m, 1055 w, 1033 w, 754 m \mathrm{~cm}^{-1}$; UV-vis $\left(\mathrm{CHCl}_{3}\right): \lambda(\log \varepsilon)=298$ (4.06), 354 (4.03) $\mathrm{nm}$;

${ }^{1} \mathrm{H}$ NMR (400 MHz, DMSO-d 6 ): $\delta=10.55(s, 1 \mathrm{H}$, $\mathrm{NH}), 7.42$ ( $d d d, J=8.2,2.6,1.2 \mathrm{~Hz}, 3 \mathrm{H}, 2^{\prime \prime}-\mathrm{H}+3_{\mathrm{a}}{ }^{\prime \prime}-$ $\mathrm{H}), 7.32\left(d d, J=8.5,6.9 \mathrm{~Hz}, 2 \mathrm{H}, 3 \mathrm{~b}^{\prime \prime}-\mathrm{H}+3^{\prime}-\mathrm{H}\right), 7.25$ $7.20\left(m, 1 \mathrm{H}, 4^{\prime \prime}-\mathrm{H}\right), 7.16(d d, J=15.3,1.5 \mathrm{~Hz}, 1 \mathrm{H}$, $\left.5^{\prime}-\mathrm{H}\right), 7.15(s, 1 \mathrm{H}, 7-\mathrm{H}), 6.96(t d, J=7.5,1.1 \mathrm{~Hz}, 1 \mathrm{H}$, $\left.4^{\prime}-\mathrm{H}\right), 6.93(s, 1 \mathrm{H}, 4-\mathrm{H}), 6.92-6.89\left(m, 1 \mathrm{H}, 6^{\prime}-\mathrm{H}\right)$, $3.87(s, 3 \mathrm{H}, 9-\mathrm{H}), 3.77(s, 3 \mathrm{H}, 10-\mathrm{H}), 3.58(s, 1 \mathrm{H}, 2-$ $\mathrm{H}), 3.52\left(d, J=17.0 \mathrm{~Hz}, 1 \mathrm{H}, 3_{\mathrm{a}}-\mathrm{H}\right), 3.04(d, J=17.0$ $\left.\mathrm{Hz}, 1 \mathrm{H}, 3_{\mathrm{b}}-\mathrm{H}\right) \mathrm{ppm} ;{ }^{13} \mathrm{C}$ NMR (100 MHz; DMSO-d 6 ): $\delta=197.4\left({\mathrm{C}-7^{\prime}}^{\prime}\right.$ C C-1), $167.9(\mathrm{C}-8), 155.5(\mathrm{C}-5)$, 150.0 (C-7a), 149.1 (C-6), 146.1 (C-3a), 135.8 (C-1'), 130.4 (C-2'), 128.2 (C-3'), 127.8 (C-5'), 127.1 (C$\left.1^{\prime \prime}\right), 127.0\left(\mathrm{C}-4^{\prime \prime}\right), 125.3 \quad$ (C-2'"), 124.8 (C-3"'), 122.4 (C-4'), 114.5 (C-6'), 107.8 (C-7), 104.2 (C-4), 66.0 (C-2), 56.0 (C-9), 55.6 (C-10), 32.7 (C-3) ppm; MS (ESI, $\mathrm{MeOH}): \mathrm{m} / \mathrm{z}(\%)=398.2\left(\left[\mathrm{M}+\mathrm{H}-\mathrm{H}_{2} \mathrm{O}\right]^{+}\right.$, 24), $438.0\left([\mathrm{M}+\mathrm{Na}]^{+}, 38\right), 642.5\left([3 \mathrm{M}+\mathrm{Ca}]^{2+}, 10\right)$, $852.9\left([2 \mathrm{M}+\mathrm{Na}]^{+}, 100\right)$;

analysis calcd for $\mathrm{C}_{25} \mathrm{H}_{21} \mathrm{NO}_{5}$ (415.44): $\mathrm{C} 72.28, \mathrm{H}$ 5.10, N 3.37; found C 71.98, H 5.34, N 3.11.

\section{3-Phenylpropanoic acid (9)}

Hydrogenation of cinnamic acid (1 g, $6.75 \mathrm{mmol})$ with $\mathrm{Pd} / \mathrm{C}(10 \%, 0.134 \mathrm{~g})$ in THF $(20 \mathrm{~mL})$. for $6 \mathrm{~h}$ at 75 psi followed by usual work-up gave 9 (0.91 g, $90 \%)^{15}$ as a white solid; $\mathrm{R}_{F}=0.45$ (silica gel, $n$-hexane/ethyl acetate, $4: 1) ; \mathrm{mp}=45-48^{\circ} \mathrm{C}$ (lit.: $\left.{ }^{22} 48-50^{\circ} \mathrm{C}\right)$; MS (ESI, MeOH): $\mathrm{m} / \mathrm{z}(\%)=149.1$ $\left([\mathrm{M}-\mathrm{H}]^{-}, 82\right), 321.0\left([2 \mathrm{M}-2 \mathrm{H}+\mathrm{Na}]^{-}, 100\right)$.

\section{2,3-Dihydro-1H-inden-1-one (10)}

Reaction of $9(0.5 \mathrm{~g}, \quad 3.3 \mathrm{mmol})$ in dry dichloromethane $(8 \mathrm{~mL})$ at $0^{\circ} \mathrm{C}$ with oxalyl chloride (1.12 mL, $13.2 \mathrm{mmol}$ ) and DMF (5 drops) followed by an additional reaction for $1 \mathrm{~h}$ at room temperature, usual work-up and reaction with $\mathrm{AlCl}_{3}(0.80 \mathrm{~g}, 5.98$ mmol) in dry dichloromethane $(8 \mathrm{~mL})$ as described above and re-crystallization from EtOH gave 3 $(0.23 \mathrm{~g}, 53 \%)$ as an off-white solid; $\mathrm{R}_{F}=0.48$ (silica gel, $n$-hexane/ethyl acetate, $4: 2) ; \mathrm{mp}=39-40^{\circ} \mathrm{C}$ (lit.: ${ }^{23} 38-39^{\circ} \mathrm{C}$ ); MS (ESI, MeOH): $\mathrm{m} / \mathrm{z}(\%)=133.1$ $\left([\mathrm{M}+\mathrm{H}]^{+}, 100\right), 155.0\left([\mathrm{M}+\mathrm{Na}]^{+}, 16\right)$.

\section{Methyl-1-oxo-2,3-dihydro-1H-indene-2- carboxylate (11)}

As described for the synthesis of $\mathbf{4}$, from $\mathbf{1 0}$ (3.00 g, $22.7 \mathrm{mmol})$ and $\mathrm{NaH}(2.73 \mathrm{~g}, 68.1 \mathrm{mmol}$, $60 \%$ in mineral oil)

followed by re-crystallization from EtOH, 11 $(1.38 \mathrm{~g}, 32 \%)$ was obtained as an off-white solid;

$\mathrm{R}_{F}=0.13$ (silica gel, chloroform); $\mathrm{mp}=49-51{ }^{\circ} \mathrm{C}$; IR (ATR): $v=1705 s, 1586 m, 1464 m, 1438 m, 1317 m$, $1273 m, 1208 s, 1157 s, 1095 m, 1007 m, 987 s, 951 m$, $853 m, 767 s, 735 m, 675 m, 513 m, 466 m \mathrm{~cm}^{-1}$; UV-vis $(\mathrm{MeOH}): \lambda(\log \varepsilon)=205$ (4.07), 246 (3.73), 296 (3.43) $\mathrm{nm}$;

${ }^{1} \mathrm{H}$ NMR (400 MHz, $\mathrm{CDCl}_{3}$ ): ${ }^{1} \mathrm{H}$ NMR (400 MHz, $\left.\mathrm{CDCl}_{3}\right): \delta=7.78(d, J=7.7 \mathrm{~Hz}, 1 \mathrm{H}, 7-\mathrm{H}), 7.66-7.61$ $(m, 1 \mathrm{H}, 6-\mathrm{H}), 7.51(d d, J=7.7,0.9 \mathrm{~Hz}, 1 \mathrm{H}, 4-\mathrm{H})$, 7.43-7.38 (m, 1H, 5-H), $3.80\left(s, 3 \mathrm{H}, 1^{\prime}-\mathrm{H}\right), 3.74(d d$, $J=17.2,4.0 \mathrm{~Hz}, 1 \mathrm{H}, 2-\mathrm{H}), 3.57(d d, J=17.2,4.0 \mathrm{~Hz}$, $\left.1 \mathrm{H}, 3_{\mathrm{a}}-\mathrm{H}\right), 3.38\left(d d, J=17.3,8.3 \mathrm{~Hz}, 1 \mathrm{H}, 3_{\mathrm{b}}-\mathrm{H}\right) \mathrm{ppm}$; ${ }^{13} \mathrm{C} \mathrm{NMR}\left(100 \mathrm{MHz} ; \mathrm{CDCl}_{3}\right): \delta=178.8(\mathrm{C}-1), 170.0$ (C-8), 153.7 (C-3a), 135.6 (C-6), 135.4 (C-7a), 128.0 (C-5), 126.7 (C-4), 124.9 (C-7), 53.3 (C-2), 52.9 (C-1'), 30.4 (C-3) ppm;

MS (ESI, MeOH): m/z $(\%)=191.0\left([\mathrm{M}+\mathrm{H}]^{+}, 100\right)$, $207.9\left(\left[\mathrm{M}+\mathrm{NH}_{4}\right]^{+}, 21\right), 213.1\left([\mathrm{M}+\mathrm{Na}]^{+}, 79\right), 225.8$ $\left([2 \mathrm{M}+\mathrm{Ca}+\mathrm{MeOH}]^{2+}, 23\right)$;

analysis calcd for $\mathrm{C}_{11} \mathrm{H}_{10} \mathrm{O}_{3}$ (190.20): C 69.46, H 5.30; found C 69.25 , H 5.47 .

\section{$N$-Ethyl-1-oxo-2,3-dihydro-1H-indene-2- carboxamide (12)}

Following the general procedure A, compound $\mathbf{1 2}$ $(0.04 \mathrm{~g}, 15 \%)$ was obtained as an off-white solid; $\mathrm{R}_{F}=0.08$ (silica gel, $n$-hexane/ethyl acetate, $4: 1$ ); $\mathrm{mp}=141-144^{\circ} \mathrm{C}$; IR (ATR): $v=3300 m, 1721 s$, $1632 s, 1552 s, 1462 m, 1421 m, 11361 m, 1324 m$, $1269 s, 1246 m, 1212 s, 1150 m, 1011 m, 993 m, 764 s$, $668 s, 592 m, 497 m, 465 \mathrm{sm}^{-1}$;

UV-vis $(\mathrm{MeOH}): \lambda(\log \varepsilon)=204$ (4.35), 246 (3.95), $295(3.39) \mathrm{nm}$;

${ }^{1} \mathrm{H}$ NMR $\left(400 \mathrm{MHz}, \mathrm{CDCl}_{3}\right): \delta=7.75(d, J=7.7 \mathrm{~Hz}$, $1 \mathrm{H}, 7-\mathrm{H}), 7.63(t, J=7.4 \mathrm{~Hz}, 1 \mathrm{H}, 6-\mathrm{H}), 7.52(d, \mathrm{~J}=$ $7.7 \mathrm{~Hz}, 1 \mathrm{H}, 4-\mathrm{H}), 7.38(t, J=7.4 \mathrm{~Hz}, 1 \mathrm{H}, 5-\mathrm{H}), 3.80$ $\left(d d, J=17.7,3.9 \mathrm{~Hz}, 1 \mathrm{H}, 3_{\mathrm{a}}-\mathrm{H}\right), 3.54(d d, J=8.3,4.0$ $\mathrm{Hz}, 1 \mathrm{H}, 2-\mathrm{H}), 3.40-3.35(m, 1 \mathrm{H}, 3 \mathrm{~b}-\mathrm{H}), 3.35-3.30(m$, $\left.2 \mathrm{H}, 1^{\prime}-\mathrm{H}\right), 1.19\left(t, J=7.3 \mathrm{~Hz}, 3 \mathrm{H}, 2^{\prime}-\mathrm{H}\right) \mathrm{ppm} ;{ }^{13} \mathrm{C}$ NMR (125 MHz; $\mathrm{CDCl}_{3}$ ): $\delta=203.8$ (C-1), 166.3 (C- 
8), 154.5 (C-3a), 135.9 (C-6), 135.6 (C-7a), 127.7 (C-5), 126.9 (C-4), 124.5 (C-7), 52.99 (C-2), 34.9 (C-1'), 28.9 (C-3), 14.9 (C-2') ppm;

MS (ESI, $\mathrm{MeOH}): \mathrm{m} / \mathrm{z}(\%)=204.1\left([\mathrm{M}+\mathrm{H}]^{+}, 100\right)$, $226.1\left([\mathrm{M}+\mathrm{Na}]^{+}, 81\right), 230.8\left([2 \mathrm{M}+\mathrm{Na}+\mathrm{H}+\mathrm{MeOH}]^{2+}\right.$, 16), $238.8\left([2 \mathrm{M}+\mathrm{Ca}+\mathrm{MeOH}]^{2+}, 27\right)$;

analysis calcd for $\mathrm{C}_{12} \mathrm{H}_{13} \mathrm{NO}_{2}$ (203.24): C 70.92, H 6.45, N 6.89; found C 70.77, H 6.61, N 6.60.

\section{$\mathrm{N}$-Butyl-1-oxo-2,3-dihydro-1H-indene-2- carboxamide (13)}

Following the general procedure A, compound $\mathbf{1 3}$ $(0.04 \mathrm{~g}, 13 \%)$ was obtained as a white solid; $\mathrm{R}_{F}=0.08$ (silica gel, $n$-hexane/ethyl acetate, $4: 1$ );

$\mathrm{mp}=112-113^{\circ} \mathrm{C}$;

IR (ATR): $v=3311 w, 2929 w, 1715 s, 1634 s, 1538 s$, $1463 m, 1423 w, 1357 m, 1326 m, 1273 m, 1211 m$, $1150 w, 1061 m, 773 m, 671 s, 594 m, 467 m \mathrm{~cm}^{-1}$; UVvis $(\mathrm{MeOH}): \lambda(\log \varepsilon)=204$ (4.18), 246 (3.77), 296 (3.20) nm;

${ }^{1} \mathrm{H}$ NMR $\left(500 \mathrm{MHz}, \mathrm{CDCl}_{3}\right): \quad \delta=7.75(d, J=7.7$ $\mathrm{Hz}, 1 \mathrm{H}, 7-\mathrm{H}), 7.63(t d, J=7.5,1.2 \mathrm{~Hz}, 1 \mathrm{H}, 6-\mathrm{H})$, 7.53-7.51 ( $m, 1 \mathrm{H}, 4-\mathrm{H}), 7.40-7.37$ ( $m, 1 \mathrm{H}, 5-\mathrm{H}), 3.80$ $\left(d d, J=17.8,4.1 \mathrm{~Hz}, 1 \mathrm{H}, 3_{\mathrm{a}}-\mathrm{H}\right), 3.54(d d, J=8.3,4.0$ $\mathrm{Hz}, 1 \mathrm{H}, 2-\mathrm{H}), 3.39-3.34\left(m, 1 \mathrm{H}, 3_{\mathrm{b}}-\mathrm{H}\right), 3.34-3.29(m$, $\left.2 \mathrm{H}, 1^{\prime}-\mathrm{H}\right), 1.57-1.51\left(\mathrm{~m}, 2 \mathrm{H}, 2^{\prime}-\mathrm{H}\right), 1.42-1.34(m$, $\left.2 \mathrm{H}, 3^{\prime}-\mathrm{H}\right), 0.94\left(t, J=7.3 \mathrm{~Hz}, 3 \mathrm{H}, 4^{\prime}-\mathrm{H}\right) \mathrm{ppm}$;

${ }^{13} \mathrm{C} \mathrm{NMR}\left(125 \mathrm{MHz} ; \mathrm{CDCl}_{3}\right): \delta=201.0(\mathrm{C}-1), 166.4$ (C-8), 154.5 (C-3a), 135.9 (C-6), 135.6 (C-7a), 127.7 (C-5), 126.9 (C-4), 124.5 (C-7), 53.0 (C-2), 39.7 (C-1'), 31.7 (C-2'), 28.9 (C-3), 20.3 (C-3'), 13.9 (C-4') ppm;

MS (ESI, $\mathrm{MeOH}): \mathrm{m} / \mathrm{z}(\%)=232.1\left([\mathrm{M}+\mathrm{H}]^{+}, 100\right)$, $254.1\left([\mathrm{M}+\mathrm{Na}]^{+}, 77\right), 258.8\left([2 \mathrm{M}+\mathrm{Na}+\mathrm{H}+\mathrm{MeOH}]^{2+}\right.$, 16), $266.8\left([2 \mathrm{M}+\mathrm{Ca}+\mathrm{MeOH}]^{2+}, 38\right)$;

analysis calcd for $\mathrm{C}_{14} \mathrm{H}_{17} \mathrm{NO}_{2}$ (231.39): $\mathrm{C} 72.70, \mathrm{H}$ 7.41, N 6.06; found C 72.51, H 7.96, N 5.81.

\section{$\mathrm{N}$-Hexyl-1-oxo-2,3-dihydro-1H-indene-2-} carboxamide (14)

Following the general procedure A, compound $\mathbf{1 4}$ $(0.03 \mathrm{~g}, 8 \%)$ was obtained as a white solid; $\mathrm{R}_{F}=0.13$ (silica gel, $n$-hexane/ethyl acetate, 4:1); $\mathrm{mp}=103-105^{\circ} \mathrm{C}$;

IR (ATR): $v=3318 w, 2926 w, 1714 s, 1633 s, 1532 s$, $1462 m, 1422 w, 1326 m, 1273 s, 1211 m, 1011 m, 773 m$, $670 s, 592 \mathrm{~m}, 467 \mathrm{~m} \mathrm{~cm}^{-1}$; UV-vis $(\mathrm{MeOH}): \lambda(\log \varepsilon)=$ 204 (4.41), 246 (3.99), 295 (3.46), 342 (3.24) nm;

${ }^{1} \mathrm{H}$ NMR $\left(500 \mathrm{MHz}, \mathrm{CDCl}_{3}\right): \delta=7.75(d, J=7.7 \mathrm{~Hz}$, $1 \mathrm{H}, 7-\mathrm{H}), 7.63(d t, J=7.6,1.1 \mathrm{~Hz}, 1 \mathrm{H}, 6-\mathrm{H}), 7.52(d t$, $J=7.7,0.9 \mathrm{~Hz}, 1 \mathrm{H}, 4-\mathrm{H}), 7.40-7.36(m, 1 \mathrm{H}, 5-\mathrm{H})$, $3.80\left(d d, J=17.8,4.0 \mathrm{~Hz}, 1 \mathrm{H}, 3_{\mathrm{a}}-\mathrm{H}\right), 3.54(d d, J=8.4$, $4.0 \mathrm{~Hz}, 1 \mathrm{H}, 2-\mathrm{H}), 3.39-3.34\left(m, 1 \mathrm{H}, 3_{\mathrm{b}}-\mathrm{H}\right), 3.34-3.27$ $\left(m, 2 \mathrm{H}, 1^{\prime}-\mathrm{H}\right), 1.58-1.52\left(m, 2 \mathrm{H}, 2^{\prime}-\mathrm{H}\right), 1.39-1.28(m$, $\left.6 \mathrm{H}, 3^{\prime}-\mathrm{H}+4^{\prime}-\mathrm{H}+5^{\prime}-\mathrm{H}\right), 0.92-0.85\left(m, 3 \mathrm{H}, 6^{\prime}-\mathrm{H}\right)$ ppm; ${ }^{13} \mathrm{C}$ NMR $\left(125 \mathrm{MHz} ; \mathrm{CDCl}_{3}\right): \delta=202.6(\mathrm{C}-1)$, 166.4 (C-8), 154.5 (C-3a), 135.9 (C-6), 135.6 (C-7a), 127.7 (C-5), 126.9 (C-4), 124.5 (C-7), 53.0 (C-2), 40.1 (C-1'), 31.6 (C-2'), 29.6 (C-3), 28.9 (C-3'), 26.7 (C-4'), 22.7 (C-5'), 14.2 (C-6') ppm;

MS (ESI, MeOH): m/z $(\%)=260.1\left([\mathrm{M}+\mathrm{H}]^{+}, 100\right)$, $282.2\left([\mathrm{M}+\mathrm{Na}]^{+}, 77\right), 286.9\left([2 \mathrm{M}+\mathrm{Na}+\mathrm{H}+\mathrm{MeOH}]^{2+}\right.$, 19), $294.8\left([2 \mathrm{M}+\mathrm{Ca}+\mathrm{MeOH}]^{2+}, 32\right)$; analysis calcd for $\mathrm{C}_{16} \mathrm{H}_{21} \mathrm{NO}_{2}$ (259.35): $\mathrm{C} 74.10, \mathrm{H}$ 8.16, N 5.40; found C 73.87, H 8.23, N 5.18.

\section{General procedure B}

Chloral hydrate (1.1 eq.) was solved in water $(2.5 \mathrm{~mL} / \mathrm{mmol})$ and heated to $35^{\circ} \mathrm{C}$. Sodium sulfate (8.9 eq.) was added by portions and stirred until the solution became clear. The corresponding aniline ( 1 eq.) was suspended in water $(0.7 \mathrm{~mL} / \mathrm{mmol})$ and added to this solution. Hydrochloric acid (36\%, 3.5 eq.) was added dropwise and a white precipitate was formed. A solution of hydroxyl ammonium chloride (3.2 eq.) in water $(1 \mathrm{~mL} / \mathrm{mmol})$ was added; this mixture was heated to $80^{\circ} \mathrm{C}$ until the reaction was completed (as indicated by TLC) ${ }^{5}$. The precipitate was filtrated off at $50^{\circ} \mathrm{C}$, washed with water and dried in vacuum. Sulfuric acid (98\%, 74 eq.) was heated to $50^{\circ} \mathrm{C}$, and the solid was added in several portions. After completion of the reaction, the mixture was poured onto ice (ca. $600 \mathrm{~mL}$ ). The precipitate was filtrated off and washed with water. To separate the 4- and 6-isomers, the solid was solved in sodium hydroxide solution $(10 \%)$ at $60^{\circ} \mathrm{C}$ and neutralized with acetic acid until $\mathrm{pH}=5$. The 4-isomer was crystallized at $5^{\circ} \mathrm{C}$ and was filtered off and washed with water. The 6-isomer crystallized after adjusting the $\mathrm{pH}=1$ by adding concentrated $\mathrm{HCl}$ and standing at $5^{\circ} \mathrm{C}$.

Compounds 15a-r were prepared according to general procedure B.

\section{4-Chloroisatin (15a)}

Compound 15a (2.00 g, 36\%) was obtained as an orange solid; $\mathrm{R}_{F}=0.17$ (silica gel, toluene/ $n$ heptane/ethyl acetate/formic acid, 80:20:10:3); $\mathrm{mp}=250-255^{\circ} \mathrm{C}$ (lit.: $\left.{ }^{24} 251^{\circ} \mathrm{C}\right)$;

MS (ESI-MeOH): m/z $(\%)=180.8\left([\mathrm{M}+\mathrm{H}]^{+}, 6\right)$, $199.0\left(\left[\mathrm{M}+\mathrm{NH}_{4}\right]^{+}, 29\right), 204.1\left([\mathrm{M}-\mathrm{Na}]^{+}, 100\right), 230.9$ $\left(\left[\mathrm{M}+\mathrm{NH}_{4}+\mathrm{MeOH}\right]^{+}, 38\right), 236.0\left([\mathrm{M}+\mathrm{Na}+\mathrm{MeOH}]^{+}\right.$, 62), $384.8 \quad\left([2 \mathrm{M}+\mathrm{Na}]^{+}, \quad 28\right), \quad 416.7$ $\left([2 \mathrm{M}+\mathrm{Na}+\mathrm{MeOH}]^{+}, 14\right), 448.9\left([2 \mathrm{M}+\mathrm{Na}+2 \mathrm{MeOH}]^{+}\right.$, 6), $180.0\left([\mathrm{M}-\mathrm{H}]^{-}, 100\right), 211.9\left([\mathrm{M}-\mathrm{H}+\mathrm{MeOH}]^{-}, 11\right)$, $215.9\left(\left[\mathrm{M}+{ }^{35} \mathrm{Cl}\right]-, 29\right), 360.7([2 \mathrm{M}-\mathrm{H}]-3), 392.8$ ([2M-H+MeOH]$\left.]^{-}, 10\right)$.

\section{6-Chloroisatin (15b)}

Compound 15b (1.40 g, 25\%) was obtained as an orange solid; $\mathrm{R}_{F}=0.27$ (silica gel, toluene $/ n$ heptane/ethyl acetate/formic acid, 80:20:10:3); $\mathrm{mp}=260-263^{\circ} \mathrm{C}$ (lit.: ${ }^{25} 261-262^{\circ} \mathrm{C}$ );

MS (ESI, MeOH): m/z $(\%)=198.9\left(\left[\mathrm{M}+\mathrm{NH}_{4}\right]^{+}, 42\right)$, $204.0\left([\mathrm{M}+\mathrm{Na}]^{+}, 100\right), 230.9\left(\left[\mathrm{M}+\mathrm{NH}_{4}+\mathrm{MeOH}\right]^{+}\right.$, 69), $236.0\left([\mathrm{M}+\mathrm{Na}+\mathrm{MeOH}]^{+}, 64\right), 384.9\left([2 \mathrm{M}+\mathrm{Na}]^{+}\right.$, 62), $416.7\left([2 \mathrm{M}+\mathrm{Na}+\mathrm{MeOH}]^{+}, 51\right), 180.0\left([\mathrm{M}-\mathrm{H}]^{-}\right.$, 100), 211.9 ([M-H+MeOH]-, 6)

\section{4-Iodoisatin (15c)}

Compound 15c (6.06 g, 72\%) was obtained as a reddish-brown solid; $\mathrm{R}_{F}=0.20$ (toluene/ $n$ heptane/ethyl acetate/formic acid, 80:20:10:3); $\mathrm{mp}=236-240^{\circ} \mathrm{C}$;

IR (KBr): $v=3564 m, 3492 m, 3454 m, 3274 s, 3078 m$, $3040 m, 2976 m, 2910 w, 2858 w, 2820 w, 2768 w$, $2696 w, 2622 w, 1742 v s, 1724 v s, 1634 m, 1606 v s$, 
$1578 v s, 1472 m, 1436 s, 1316 m, 1274 m, 1242 s, 1194 w$, $1158 s, 1136 m, 1054 w, 1022 w, 900 m, 792 m, 664 m$, $646 m \mathrm{~cm}^{-1}$;

UV-vis $(\mathrm{MeOH}): \lambda(\log \varepsilon)=229$ (4.25), $326(3.55)$ $\mathrm{nm} ;{ }^{1} \mathrm{H}$ NMR $\left(500 \mathrm{MHz}, \mathrm{DMSO}-\mathrm{d}_{6}\right): \delta=11.06(s, 1 \mathrm{H}$, $\mathrm{NH}), 7.48(d d, J=7.9,0.7 \mathrm{~Hz}, 1 \mathrm{H}, 5-\mathrm{H}), 7.25(t, J=$ $7.9 \mathrm{~Hz}, 1 \mathrm{H}, 6-\mathrm{H}), 6.90(d d, J=7.8,0.7 \mathrm{~Hz}, 1 \mathrm{H}, 7-\mathrm{H})$ ppm; ${ }^{13} \mathrm{C}$ NMR (100 MHz, DMSO-d 6 ): $\delta=183.6$ (C-3), 159.3 (C-2), 154.1 (C-8), 138.4 (C-6), 132.8 (C-5), 119.3 (C-3a), 112.0 (C-7), 92.9 (C-4) ppm; MS (ESI, MeOH): m/z $(\%)=274.0\left([\mathrm{M}+\mathrm{H}]^{+}, 5\right)$, $290.8\left(\left[\mathrm{M}+\mathrm{NH}_{4}\right]^{+}, 37\right), 296.0\left([\mathrm{M}+\mathrm{Na}]^{+}, 100\right), 306.0$ $\left([\mathrm{M}+\mathrm{H}+\mathrm{MeOH}]^{+}, \quad 15\right), 323.0\left(\left[\mathrm{M}+\mathrm{NH}_{4}+\mathrm{MeOH}\right]^{+}\right.$, $41), 327.5\left([\mathrm{M}+\mathrm{Na}+\mathrm{MeOH}]^{+}, 59\right), 568.6\left([2 \mathrm{M}+\mathrm{Na}]^{+}\right.$, $80), \quad 600.6 \quad\left([2 \mathrm{M}+\mathrm{Na}+\mathrm{MeOH}]^{+}, \quad 22\right), \quad 632.7$ $\left([2 \mathrm{M}+\mathrm{Na}+2 \mathrm{MeOH}]^{+}, 9\right), 272.0\left([\mathrm{M}-\mathrm{H}]^{-}, 100\right), 303.8$ $\left([\mathrm{M}-\mathrm{H}+\mathrm{MeOH}]^{-}, 19\right), 307.8\left([\mathrm{M}+\mathrm{Cl}]^{-}, 52\right), 544.5$ ([2M-H]-, 35);

analysis calcd for $\mathrm{C}_{8} \mathrm{H}_{4} \mathrm{INO}_{2}$ (273.03): C 35.19, H 1.48, N 5.13; found C 34.77, H 1.72, N 4.90.

\section{6-Iodoisatin (15d)}

Compound 15d (0.75 g, 9\%) was obtained as an orange solid; $\mathrm{R}_{F}=0.33$ (silica gel, toluene $/ n$ heptane/ethyl acetate/formic acid, 80:20:10:3); $\mathrm{mp}=267-272^{\circ} \mathrm{C}$;

IR (KBr): $v=3508 m, 3462 m, 3190 m, 3166 m, 3094 w$, $3064 w, 3022 w, 2850 w, 2802 w, 2362 w, 2342 w, 1744 s$, $1730 v s, 1610 v s, 1542 w, 1508 w, 1474 w, 1458 w$, $1434 m, 1384 w, 1368 w, 1326 m, 1270 w, 1250 w$, $1196 \mathrm{~m}, 1186 w, 1130 w, 1102 \mathrm{~m}, 1048 \mathrm{~m} \mathrm{~cm}^{-1}$;

UV-vis $(\mathrm{MeOH}): \lambda(\log \varepsilon)=217$ (4.07), 236 (4.07), 259 (4.07), 316 (4.07), 402 (3.37) nm; ${ }^{1} \mathrm{H}$ NMR (400 MHz, DMSO-d $\left.\mathrm{d}_{6}\right): \delta=11.19(s, 1 \mathrm{H}, \mathrm{NH}), 7.47(d, J=$ $6.3 \mathrm{~Hz}, 1 \mathrm{H}, 4-\mathrm{H}), 7.30(s, 1 \mathrm{H}, 7-\mathrm{H}), 7.24(d d, J=7.5$, $1.0 \mathrm{~Hz}, 1 \mathrm{H}, 5-\mathrm{H}) \mathrm{ppm} ;{ }^{13} \mathrm{C}$ NMR (125 MHz, DMSO$\left.\mathrm{d}_{6}\right): \delta=183.6(\mathrm{C}-3), 159.0(\mathrm{C}-2), 151.1(\mathrm{C}-7 \mathrm{a}), 131.5$ (C-5), 125.6 (C-4), 120.6 (C-7), 117.2 (C-3a), 107.1 (C-6) ppm;

MS (ESI, $\mathrm{MeOH}): \mathrm{m} / \mathrm{z}(\%)=272.0\left([\mathrm{M}-\mathrm{H}]^{-}, 100\right)$, $289.9\left(\left[\mathrm{M}-\mathrm{H}+\mathrm{H}_{2} \mathrm{O}\right]^{-}, 4\right), 303.8\left([\mathrm{M}-\mathrm{H}+\mathrm{MeOH}]^{-}, 6\right)$, $307.7\left(\left[\mathrm{M}+{ }^{35} \mathrm{Cl}\right]-, 2\right)$;

analysis calcd for $\mathrm{C}_{8} \mathrm{H}_{4} \mathrm{INO}_{2}$ (273.03): C 35.19, H 1.48, N 5.13; found C 34.96, H 1.69, N 4.93

\section{7-Chloroisatin (15e)}

Compound 15e $(0.52 \mathrm{~g}, 94 \%)$ was obtained as a reddish-brown solid; $\mathrm{R}_{F}=0.37$ (silica gel, toluene/ $n$ heptane/ethyl acetate/formic acid, 80:20:10:3); $\mathrm{mp}=182-185^{\circ} \mathrm{C}$ (lit.: $\left.{ }^{26} 184-186^{\circ} \mathrm{C}\right)$;

MS (ESI, MeOH): m/z $(\%)=199.0\left(\left[\mathrm{M}+\mathrm{NH}_{4}\right]^{+}, 17\right)$, $204.0\left([\mathrm{M}+\mathrm{Na}]^{+}, 93\right), 230.9\left(\left[{ }^{35} \mathrm{M}+\mathrm{NH}_{4}+\mathrm{MeOH}\right]^{+}\right.$, 40), $236.0\left([\mathrm{M}+\mathrm{Na}+\mathrm{MeOH}]^{+}, 100\right), 180.0\left(\left[{ }^{35} \mathrm{M}-\mathrm{H}\right]^{-}\right.$, $100)$.

\section{7-Iodoisatin (15f)}

Compound 15f (2.97 g, 71\%) was afforded as a brownish solid; $\mathrm{R}_{F}=0.43$ (silica gel, toluene $/ n$ heptane/ethyl acetate/formic acid, 80:20:10:3); $\mathrm{mp}=267-272^{\circ} \mathrm{C}$;

IR (KBr): $v=3446 w, 3208 m, 3178 m, 3098 w, 3064 w$, $2852 w, 2772 w, 2594 w, 1740 v s, 1646 w, 1604 v s$, $1540 w, 1472 m, 1426 m, 1382 w, 1320 s, 1280 w, 1262 w$,
$1218 m, 1198 m, 1174 m, 1116 m, 1082 w, 1052 w, 956 m$, $764 m \mathrm{~cm}^{-1}$.

UV-vis $(\mathrm{MeOH}): \lambda(\log \varepsilon)=225 \mathrm{~nm}(4.15), 305 \mathrm{~nm}$ (3.45), $405 \mathrm{~nm}$ (3.45);

${ }^{1} \mathrm{H}$ NMR (400 MHz, DMSO-d 6$): \delta=11.00(s, 1 \mathrm{H}$, $\mathrm{NH}), 7.94(d d, J=7.9,1.0 \mathrm{~Hz}, 1 \mathrm{H}, 6-\mathrm{H}), 7.50(d, J=$ $7.3 \mathrm{~Hz}, 1 \mathrm{H}, 4-\mathrm{H}), 6.89(t, J=7.7 \mathrm{~Hz}, 1 \mathrm{H}, 5-\mathrm{H}) \mathrm{ppm}$; ${ }^{13} \mathrm{C}$ NMR (100 MHz, DMSO-d $\left.{ }_{6}\right): \delta=184.3(\mathrm{C}-3)$, 159.8 (C-2), 152.9 (C-7a), 146.4 (C-6), 124.5 (C-4), 123.9 (C-5), 119.6 (C-3a), 78.3 (C-7) ppm;

MS (ESI, $\mathrm{MeOH}): \mathrm{m} / \mathrm{z}(\%)=290.9\left(\left[\mathrm{M}+\mathrm{NH}_{4}\right]^{+}, 30\right)$, $296.0\left([\mathrm{M}+\mathrm{Na}]^{+}, 100\right), 323.0\left(\left[\mathrm{M}+\mathrm{NH}_{4}+\mathrm{MeOH}\right]^{+}\right.$, 37), $328.0\left([\mathrm{M}+\mathrm{Na}+\mathrm{MeOH}]^{+}, 72\right), 568.5\left([2 \mathrm{M}+\mathrm{Na}]^{+}\right.$, $50), \quad 600.6 \quad\left([2 \mathrm{M}+\mathrm{Na}+\mathrm{MeOH}]^{+}, \quad 37\right), \quad 632.4$ $\left([2 \mathrm{M}+\mathrm{Na}+2 \mathrm{MeOH}]^{+}, 8\right), 272.0\left([\mathrm{M}-\mathrm{H}]^{-}, 100\right), 303.9$ ([M-H+MeOH]-, 20);

analysis calcd for $\mathrm{C}_{8} \mathrm{H}_{4} \mathrm{INO}_{2}$ (273.03): C 35.19, H 1.48, N 5.13; found C 35.01, H 1.61, N 4.94.

\section{6-Fluoroisatin (15g)}

Compound 15g (2.93 g, 59\%) was obtained as a yellow solid; $\mathrm{R}_{F}=0.29$ (silica gel, toluene $/ n$ heptane/ethyl acetate/formic acid, 80:20:10:3); mp = 197-199 ${ }^{\circ} \mathrm{C}$ (lit.: ${ }^{27} 195-196^{\circ} \mathrm{C}$ );

MS (ESI, $\mathrm{MeOH}): \mathrm{m} / \mathrm{z}(\%)=166.1\left([\mathrm{M}+\mathrm{H}]^{+}, 68\right)$, $188.0\left([\mathrm{M}+\mathrm{Na}]^{+}, 84\right), 197.8\left([\mathrm{M}+\mathrm{H}+\mathrm{MeOH}]^{+}, 100\right)$, $220.0 \quad\left([\mathrm{M}+\mathrm{Na}+\mathrm{MeOH}]^{+}, \quad 24\right), \quad 228.8$ $\left([\mathrm{M}+\mathrm{Na}+\mathrm{MeCN}]^{+}, \quad 98\right), \quad 260.6$ $\left([\mathrm{M}+\mathrm{Na}+\mathrm{MeOH}+\mathrm{MeCN}]^{+}, 21\right), 352.8\left([2 \mathrm{M}+\mathrm{Na}]^{+}\right.$, 61), $164\left([\mathrm{M}-\mathrm{H}]^{-}, 100\right)$.

\section{7-Fluoroisatin (15h)}

Compound 15h (1.31 g, 51\%) was obtained as a brown solid; $\mathrm{R}_{F}=0.23$ (silica gel, toluene $/ n$ heptane/ethyl acetate/formic acid, 80:20:10:3);

$\mathrm{mp}=193-195^{\circ} \mathrm{C}$;

IR (KBr): $v=3440 m, 3424 m, 3196 m, 3102 m, 3058 m$, $1742 v s, 1638 v s, 1602 m, 1560 w, 1540 w, 1496 s$, $1454 m, 1404 w, 1326 s, 1286 m, 1260 s, 1228 m, 1206 s$, $1158 m, 1114 w, 1080 w, 1054 m, 1034 m, 1002 m, 778 m$, $704 m, 582 \mathrm{~m} \mathrm{~cm}^{-1}$;

UV-vis $(\mathrm{MeOH}): \lambda(\log \varepsilon)=206$ (3.66), 237 (3.66), 294 (3.26) nm;

${ }^{1} \mathrm{H}$ NMR (400 MHz, DMSO-d 6 ): $\delta=11.52(s, 1 \mathrm{H}$, $\mathrm{NH}), 7.52$ ( $d d d, J=10.4,8.3,1.0 \mathrm{~Hz}, 1 \mathrm{H}, 6-\mathrm{H}), 7.36$ $(d d d, J=7.4,1.7,0.8 \mathrm{~Hz}, 1 \mathrm{H}, 4-\mathrm{H}), 7.06(d d d, J=8.3$, 7.5, $4.3 \mathrm{~Hz}, 1 \mathrm{H}, 5-\mathrm{H}) \mathrm{ppm} ;{ }^{13} \mathrm{C} \mathrm{NMR}(125 \mathrm{MHz}$, DMSO-d $)): \delta=183.2(d, J=4.3 \mathrm{~Hz}, \mathrm{C}-3), 159.2(s$, C-2), $147.2(d, J=245.2 \mathrm{~Hz}, \mathrm{C}-7), 137.4(d, J=13.3$ $\mathrm{Hz}, \mathrm{C}-7 \mathrm{a}), 124.7$ ( $d, J=17.5 \mathrm{~Hz}, \mathrm{C}-6), 123.4(d, J=$ $5.4 \mathrm{~Hz}, \mathrm{C}-5), 120.6(d, J=3.3 \mathrm{~Hz}, \mathrm{C}-4), 120.5(d, J=$ $3.9 \mathrm{~Hz}, \mathrm{C}-3 \mathrm{a}) \mathrm{ppm} ;{ }^{19} \mathrm{~F}$ NMR (470 MHz, DMSO-d 6 ): $\delta=-133.06(d d, J=10.5,4.3 \mathrm{~Hz}) \mathrm{ppm}$;

MS (ESI, MeOH): $\mathrm{m} / \mathrm{z}(\%)=166.4\left([\mathrm{M}+\mathrm{H}]^{+}, 7\right)$, $188.1\left([\mathrm{M}+\mathrm{Na}]^{+}, 75\right), 220.1\left([\mathrm{M}+\mathrm{Na}+\mathrm{MeOH}]^{+}, 58\right)$, $164.0\left([\mathrm{M}-\mathrm{H}]^{-}, 28\right)$;

analysis calcd for $\mathrm{C}_{8} \mathrm{H}_{4} \mathrm{FNO}_{2}$ (165.12): C 58.19, H 2.44, N 8.48; found C 57.86, H 2.63, N 8.52.

\section{6,7-Difluoroisatin (15i)}

Compound 15i (2.69 g, 92\%) was obtained as an orange solid; $\mathrm{R}_{F}=0.41$ (silica gel, toluene $/ n$ heptane/ethyl acetate/formic acid, 80:20:10:3); $\mathrm{mp}=154-159^{\circ} \mathrm{C}$; 
IR (KBr): $v=3466 m, 3428 w, 3182 s, 3122 m, 3078 m$, $2824 m, 2640 w, 1758 v s, 1742 v s, 1702 m, 1638 v s$, $1612 s, 1558 m, 1520 v s, 1458 s, 1418 m, 1398 m, 1384 m$, $1342 v s, 1292 s, 1272 s, 1248 s, 1224 m, 1198 s, 1158 s$, $1090 m, 1044 s, 992 m, 936 m, 902 s, 870 m, 842 s, 796 m$, $786 m, 744 m, 704 s, 654 s, 606 s, 544 m \mathrm{~cm}^{-1}$;

UV-vis $(\mathrm{MeOH}): \lambda(\log \varepsilon)=209$ (3.89), 239 (3.89), 294 (3.19), 296 (3.89), 390 (3.19) nm;

${ }^{1} \mathrm{H}$ NMR (400 MHz, DMSO-d 6 ): $\delta=11.80(s, 1 \mathrm{H}$, $\mathrm{NH}), 7.45$ ( $d d, J=8.3,4.8 \mathrm{~Hz}, 1 \mathrm{H}, 4-\mathrm{H}), 7.08$ (ddd, $J$ $=11.1,8.2,6.9 \mathrm{~Hz}, 1 \mathrm{H}, 5-\mathrm{H}) \mathrm{ppm} ;{ }^{13} \mathrm{C} \mathrm{NMR}(125$ MHz, DMSO-d $\left.\mathrm{d}_{6}\right): \delta=181.6(d, J=3.7 \mathrm{~Hz}, \mathrm{C}-3)$, $159.4(\mathrm{C}-2), 155.7(d d, J=255.4,10.0 \mathrm{~Hz}, \mathrm{C}-6), 139.6$ $(d d, J=9.5,4.9 \mathrm{~Hz}, \mathrm{C}-7 \mathrm{a}), 135.8(d d, J=249.0,16.9$ $\mathrm{Hz}, \mathrm{C}-7), 122.2(d d, J=10.0,3.7 \mathrm{~Hz} \mathrm{C}-4), 116.4(t, J$ $=2.8 \mathrm{~Hz}, \mathrm{C}-3 \mathrm{a}), 110.6(d, J=19.5 \mathrm{~Hz}, \mathrm{C}-5) \mathrm{ppm} ;{ }^{19} \mathrm{~F}$ NMR (470 MHz, DMSO-d 6 ): $\delta=-124.45$ (ddd, $J=$ $\left.21.0,11.1,4.8 \mathrm{~Hz}, \mathrm{~F}_{6}\right),-157.62(d d, J=20.9,6.6, \mathrm{~Hz}$, $\left.\mathrm{F}_{7}\right) \mathrm{ppm}$;

MS (ESI, $\mathrm{MeOH}): \mathrm{m} / \mathrm{z}(\%)=206.0\left([\mathrm{M}+\mathrm{Na}]^{+}, 46\right)$, $237.9\left([\mathrm{M}+\mathrm{MeOH}]^{+}, 24\right), 388.8\left([2 \mathrm{M}+\mathrm{Na}]^{+}, 13\right)$, 182.1 ([M-H] $\left.]^{-}, 100\right), 213.9$ ([M-H+MeOH] $\left.]^{-}, 11\right)$; analysis calcd for $\mathrm{C}_{8} \mathrm{H}_{3} \mathrm{~F}_{2} \mathrm{NO}_{2}$ (183.11): C 52.47, H 1.65, N 7.65; found C 52.21, H 1.79, N 7.50.

\section{4,5-Dimethylisatin (15j)}

Compound 15j (1.05 g, 21\%) was obtained as a reddish-brown solid; $\mathrm{R}_{F}=0.23$ (silica gel, toluene $/ n$ heptane/ethyl acetate/formic acid, 80:20:10:3);

$\mathrm{mp}=220-222^{\circ} \mathrm{C}$ (lit.: ${ }^{25} 217-218^{\circ} \mathrm{C}$ );

MS (ESI, MeOH): m/z $(\%)=176.1\left([\mathrm{M}+\mathrm{H}]^{+}, 27\right)$, $193.0\left(\left[\mathrm{M}+\mathrm{NH}_{4}\right]^{+}, 100\right), 198.1\left([\mathrm{M}+\mathrm{Na}]^{+}, 30\right), 372.9$ $\left([2 \mathrm{M}+\mathrm{Na}]^{+} ; 100\right), 174.1\left([\mathrm{M}-\mathrm{H}]^{-}, 100\right.$.

\section{5,6-Dimethylisatin (15k)}

Compound 15k (1.57 g, 32\%) was obtained as a red solid; $\mathrm{R}_{F}=0.21$ (silica gel, toluene/ $n$-heptane/ethyl acetate/formic acid, 80:20:10:3); $\mathrm{mp}=213-214^{\circ} \mathrm{C}$ (lit.: $\left.^{28} 214-215^{\circ} \mathrm{C}\right)$; MS (ESI, MeOH): m/z $(\%)=$ $176.1\left([\mathrm{M}+\mathrm{H}]^{+}, 13\right), 193.0\left(\left[\mathrm{M}+\mathrm{NH}_{4}\right]^{+}, 36\right), 198.1$ $\left([\mathrm{M}+\mathrm{Na}]^{+}, \quad 9\right), \quad 370.0 \quad\left(\left[2 \mathrm{M}+\mathrm{NH}_{4}\right]^{+}, \quad 14\right), \quad 372.9$ $\left([2 \mathrm{M}+\mathrm{Na}]^{+}, 100\right), 404\left([2 \mathrm{M}+\mathrm{Na}+\mathrm{MeOH}]^{+}, 6\right), 174.1$ ([M-H]', 100).

\section{4-Bromoisatin (15l)}

Compound 15l (3.93 g, 57\%) was obtained as an orange solid; $\mathrm{R}_{F}=0.20$ (silica gel, toluene $/ n$ heptane/ethyl acetate/formic acid, 80:20:10:3);

$\mathrm{mp}=266-269^{\circ} \mathrm{C}\left(\right.$ lit.: $\left.{ }^{29} 258-259^{\circ} \mathrm{C}\right)$;

MS (ESI, MeOH): m/z $(\%)=228.0\left([\mathrm{M}+\mathrm{H}]^{+}, 10\right)$, $243.0\left(\left[\mathrm{M}+\mathrm{NH}_{4}\right]^{+}, 41\right), 248.0\left([\mathrm{M}+\mathrm{Na}]^{+}, 100\right), 274.9$ $\left(\left[\mathrm{M}+\mathrm{NH}_{4}+\mathrm{MeOH}\right]^{+}, 85\right), 279.9\left([\mathrm{M}+\mathrm{Na}+\mathrm{MeOH}]^{+}\right.$, 67), $474.7 \quad\left([2 \mathrm{M}+\mathrm{Na}]^{+}, \quad 79\right), \quad 506.7$ $\left([2 \mathrm{M}+\mathrm{Na}+\mathrm{MeOH}]^{+}, 49\right), 538.7\left([2 \mathrm{M}+\mathrm{Na}+2 \mathrm{MeOH}]^{+}\right.$, 27), 226.0 ([M-H]-, 100), 255.9 ([M-H+MeOH]-, 19), $260.0\left(\left[\mathrm{M}+{ }^{35} \mathrm{Cl}\right]^{-}, 18\right), 450.5$ ([2M-H] $\left.]^{-}, 19\right)$.

\section{6-Bromoisatin (15m)}

Compound $15 \mathrm{~m}(1.74 \mathrm{~g}, 25 \%)$ was obtained as an orange solid; $\mathrm{R}_{F}=0.36$ (silica gel, toluene $/ n$ heptane/ethyl acetate/formic acid, 80:20:10:3);

$\mathrm{mp}=269-271^{\circ} \mathrm{C}$ (lit.: ${ }^{30} 272.5^{\circ} \mathrm{C}$ );

MS (ESI, $\mathrm{MeOH}): \mathrm{m} / \mathrm{z}(\%)=228.2\left([\mathrm{M}+\mathrm{H}]^{+}, 8\right)$, $242.9\left(\left[\mathrm{M}+\mathrm{NH}_{4}\right]^{+}, 46\right), 248.0\left([\mathrm{M}+\mathrm{Na}]^{+}, 100\right), 257.8$ $\left([\mathrm{M}+\mathrm{H}+\mathrm{MeOH}]^{+}, 10\right), 265.7\left(\left[\mathrm{M}+\mathrm{Na}+\mathrm{H}_{2} \mathrm{O}\right]^{+}, 12\right)$,
$274.8 \quad\left(\left[\mathrm{M}+\mathrm{NH}_{4}+\mathrm{MeOH}\right]^{+}, \quad 40\right), \quad 279.9$ $\left([\mathrm{M}+\mathrm{Na}+\mathrm{MeOH}]^{+}, 41\right), 224.0\left([\mathrm{M}-\mathrm{H}]^{-}, 100\right), 241.9$ $\left(\left[\mathrm{M}-\mathrm{H}+\mathrm{H}_{2} \mathrm{O}\right]^{-}, 11\right), 255.9$ ([M-H+MeOH] $\left.]^{-}, 4\right)$.

\section{7-Bromoisatin $(15 n)$}

Compound 15n (2.91 g, 83\%) was obtained as a reddish-brown solid; $\mathrm{R}_{F}=0.41$ (silica gel, toluene $/ n$ heptane/ethyl acetate/formic acid, 80:20:10:3); $\mathrm{mp}=196-198^{\circ} \mathrm{C}$ (lit.: $\left.{ }^{31} 195-200^{\circ} \mathrm{C}\right)$;

MS (ESI, MeOH): m/z $(\%)=243.2\left(\left[\mathrm{M}+\mathrm{NH}_{4}\right]^{+}, 17\right)$, $250.0\left([\mathrm{M}+\mathrm{Na}]^{+}, 94\right), 275.0\left(\left[\mathrm{M}+\mathrm{NH}_{4}+\mathrm{MeOH}\right]^{+}, 43\right)$, $280.0\left([\mathrm{M}+\mathrm{Na}+\mathrm{MeOH}]^{+}, 100\right), 224.0\left([\mathrm{M}-\mathrm{H}]^{-}, 100\right)$, $303.9\left(\left[\mathrm{M}+{ }^{79} \mathrm{Br}\right],-10\right)$.

\section{5-Bromoisatin (15o)}

Compound $150(0.71 \mathrm{~g}, 93 \%)$ was obtained as an orange solid; $\mathrm{R}_{F}=0.23$ (silica gel, toluene/ $n$ heptane/ethyl acetate/formic acid, 80:20:10:3);

$\mathrm{mp}=253-255^{\circ} \mathrm{C}$ (lit.: $\left.{ }^{32} 254-255^{\circ} \mathrm{C}\right)$;

ESI-MS $(\mathrm{MeOH}): \mathrm{m} / \mathrm{z}(\%)=224.0\left([\mathrm{M}-\mathrm{H}]^{-}, 83\right)$, 257.9 ([M-H+MeOH $\left.]^{-}, 7\right)$.

\section{5-Iodoisatin (15p)}

Compound 15p (0.25 g, 17\%) was obtained as a brownish solid; $\mathrm{R}_{F}=0.22$ (silica gel, toluene $/ n$ heptane/ethyl acetate/formic acid, 80:20:10:3);

$\mathrm{mp}=260-262^{\circ} \mathrm{C}$ (lit.: $\left.{ }^{26} 254-259^{\circ} \mathrm{C}\right)$;

MS (ESI, MeOH): m/z $(\%)=296.0\left([\mathrm{M}+\mathrm{Na}]^{+}, 93\right)$, $323.2 \quad\left(\left[\mathrm{M}+\mathrm{NH}_{4}+\mathrm{MeOH}\right]^{+}, \quad 47\right), \quad 327.9$ $\left([\mathrm{M}+\mathrm{Na}+\mathrm{MeOH}]^{+}, 100\right), 568.5\left([2 \mathrm{M}+\mathrm{Na}]^{+}, 22\right)$, $600.7\left([2 \mathrm{M}+\mathrm{Na}+\mathrm{MeOH}]^{+}, 11\right), 271.9\left([\mathrm{M}-\mathrm{H}]^{-}, 100\right)$, 303.8 ([M-H+MeOH]$\left.]^{-}, 26\right), 307.7\left(\left[\mathrm{M}+{ }^{35} \mathrm{Cl}\right]^{-}, 11\right)$, 544.5 ([2M-H], 26$)$.

\section{5,6-Dimethoxyisatin (15q)}

Compound 15q (2.31 g, 68\%) was obtained as a brown solid; $\mathrm{R}_{F}=0.22$ (silica gel, toluene $/ n$ heptane/ethyl acetate/formic acid, 80:20:10:3); $\mathrm{mp}=180-183^{\circ} \mathrm{C}$ (lit.: $\left.{ }^{33} 180-195^{\circ} \mathrm{C}\right)$;

MS (ESI, MeOH): m/z $(\%)=208.1\left([\mathrm{M}+\mathrm{H}]^{+}, 100\right)$, $230.1\left([\mathrm{M}+\mathrm{Na}]^{+}, 82\right), 235.7\left([2 \mathrm{M}+\mathrm{Na}+\mathrm{H}+\mathrm{MeOH}]^{2+}\right.$, 34), $242.8\left([2 \mathrm{M}+\mathrm{Ca}+\mathrm{MeOH}]^{2+}, 15\right)$.

\section{5-Nitroisatin (15r)}

Compound 15r (0.08 g, 89\%) was obtained as a yellow solid; $\mathrm{R}_{F}=0.22$ (silica gel, toluene $/ n$ heptane/ethyl acetate/formic acid, 80:20:10:3); $\mathrm{mp}=165-168^{\circ} \mathrm{C}$;

${ }^{1} \mathrm{H}$ NMR (400 MHz, DMSO-d 6 ): $\delta=11.65(s, 1 \mathrm{H}$, $\mathrm{NH}), 8.45(d d, J=8.7,2.4 \mathrm{~Hz}, 1 \mathrm{H}, 6-\mathrm{H}), 8.22(d, J=$ $2.2 \mathrm{~Hz}, 1 \mathrm{H}, 4-\mathrm{H}), 7.09$ (d, $J=8.7 \mathrm{~Hz}, 1 \mathrm{H}, 7-\mathrm{H}) \mathrm{ppm}$; ${ }^{13} \mathrm{C}$ NMR (100 MHz, DMSO-d 6 ): $\delta=182.3(\mathrm{C}-3)$, 159.8 (C-2), 155.2 (C-7a), 142.6 (C-3a), 133.0 (C-6), 119.6 (C-4), 118.1 (C-5), 112.5 (C-7) ppm;

MS (ESI, MeOH): m/z (\%) = $193.0\left([\mathrm{M}+\mathrm{H}]^{+}, 100\right)$, $215.1\left([\mathrm{M}+\mathrm{Na}]^{+}, 81\right)$; analysis calcd for $\mathrm{C}_{8} \mathrm{H}_{4} \mathrm{~N}_{2} \mathrm{O}_{4}$ (192.13): 50.01, H 2.10, N 14.58; found: C 49.73, H 2.31, N 14.37 .

\section{General procedure $\mathbf{C}$}

To a solution of the isatin derivative (1 eq.) in acetic acid $(1 \mathrm{M})$ at $0^{\circ} \mathrm{C}$, bromine (1.2 eq.) was added dropwise, so that the temperature did not $4{ }^{\circ} \mathrm{C}{ }^{6}$. The reaction mixture was stirred for $1 \mathrm{~h}$ at $0^{\circ} \mathrm{C}$ and poured on ice. The solid was filtered off, washed with water 
and dried under diminished pressure followed by column purification.

\section{5-Bromo-4-chloroisatin (16a)}

Column chromatography (silica gel, $n$-hexane/ethyl acetate, $7: 4)$ afforded $\mathbf{1 6 a}(0.15 \mathrm{~g}, 45 \%)$ as a red solid; $\mathrm{R}_{F}=0.29$ (silica gel, $n$-hexane/ethyl acetate, 7:4); $\mathrm{mp}=261-264^{\circ} \mathrm{C}$;

IR (ATR): $v=3219 w, 1737 s, 1604 s, 1480 m, 1435 m$, $1382 m, 1269 m, 1236 s, 1150 m, 1116 m, 1043 m, 841 m$, $824 m, 788 m, 688 s, 665 s, 602 s, 552 m \mathrm{~cm}^{-1}$;

UV-vis (MeOH): $\lambda(\log \varepsilon)=219$ (4.06), 250 (3.95), 309 (3.06) nm; ${ }^{1} \mathrm{H}$ NMR (400 MHz, DMSO-d 6 ): $\delta=$ $11.27(s, 1 \mathrm{H}, \mathrm{NH}), 7.89(d, J=8.4 \mathrm{~Hz}, 1 \mathrm{H}, 6-\mathrm{H}), 6.82$ $(d, J=8.4 \mathrm{~Hz}, 1 \mathrm{H}, 7-\mathrm{H}) \mathrm{ppm} ;{ }^{13} \mathrm{C} \mathrm{NMR}(100 \mathrm{MHz}$, DMSO-d $\left.{ }_{6}\right): \delta=180.3(\mathrm{C}-3), 158.3(\mathrm{C}-2), 151.1(\mathrm{C}-$ 7a), 141.3 (C-6), 130.8 (C-4), 116.4 (C-5), 115.3 (C3a), 112.5 (C-7) ppm;

MS (ESI, $\mathrm{MeOH}): \mathrm{m} / \mathrm{z}(\%)=283.9\left([\mathrm{M}+\mathrm{Na}]^{+}, 71\right)$, $308.8 \quad\left(\left[\mathrm{M}+\mathrm{NH}_{4}+\mathrm{MeOH}\right]^{+}, \quad 29\right), \quad 315.9$ $\left([\mathrm{M}+\mathrm{Na}+\mathrm{MeOH}]^{+}, 100\right), 259.9$ ([M-H] $\left.]^{-}, 100\right), 291.8$ ([M-H+MeOH]- 12);

analysis calcd for $\mathrm{C}_{8} \mathrm{H}_{3} \mathrm{BrClNO}_{2}$ (260.47): C 36.89, H 1.16, N 5.38; found C 36.50, H 1.37, N 5.06.

\section{5-Bromo-6-chloroisatin (16b)}

Column chromatography (silica gel, $n$-hexane/ethyl acetate, 7:4) afforded $\mathbf{1 6 b}(0.06 \mathrm{~g}, 18 \%)$ as an orange solid; $\mathrm{R}_{F}=0.38$ (silica gel, $n$-hexane/ethyl acetate, $7: 4) ; \mathrm{mp}=260-263^{\circ} \mathrm{C}$;

IR (ATR): $v=3276 w, 2923 w, 1768 m, 1737 s, 1602 s$, $1449 m, 1408 m, 1261 m, 1159 m, 1092 m, 972 m, 901 m$, $863 m, 702 m, 662 s, 616 s, 573 m, 457 \mathrm{sm}^{-1}$;

UV-vis $(\mathrm{MeOH}): \lambda(\log \varepsilon)=219$ (4.62), 257 (4.38), 301 (3.62) nm; ${ }^{1} \mathrm{H}$ NMR (500 MHz, DMSO-d 6 ) $\delta=$ 10.38 (s, 1H, NH), 7.00 (s, 1H, 4-H), 6.26 (s, 1H, 7H) ppm; ${ }^{13} \mathrm{C}$ NMR (125 MHz, DMSO-d 6 ): $\delta=182.1$ (C-3), 159.1 (C-2), 150.2 (C-7a), 141.5 (C-6), 129.1 (C-4), 118.4 (C-5), 114.4 (C-3a), 113.9 (C-7) ppm; MS (ESI, MeOH): m/z $(\%)=259.9\left([\mathrm{M}-\mathrm{H}]^{-} ; 100\right)$, 291.87 ([M-H+MeOH] $\left.]^{-}, 7\right)$;

analysis calcd for $\mathrm{C}_{8} \mathrm{H}_{3} \mathrm{BrClNO}_{2}$ (260.47): C 36.89, $\mathrm{H}$ 1.16, N 5.38; found C 36.57, H 1.38, N 5.11.

\section{5-Bromo-4-iodoisatin (16c)}

Column chromatography (silica gel, $n$-hexane/ethyl acetate, $7: 4)$ afforded $16 \mathrm{c}(0.17 \mathrm{~g}, 68 \%)$ as a red solid; $\mathrm{R}_{F}=0.35$ (silica gel, $n$-hexane/ethyl acetate, 7:4); $\mathrm{mp}=220-223^{\circ} \mathrm{C}$;

IR (ATR): $v=3143 w, 1738 s, 1600 s, 1570 s, 1467 m$, $1424 m, 1392 w, 1314 m, 1245 s, 1157 m, 1132 m$, $1105 m, 1051 m, 856 m, 831 w, 782 s, 773 m, 668 s, 644 m$, $560 \mathrm{~m} \mathrm{~cm}^{-1}$;

UV-vis (MeOH): $\lambda(\log \varepsilon)=205$ (4.05), 229 (4.08), 322 (2.99), 421 (2.77) nm; ${ }^{1} \mathrm{H}$ NMR (400 MHz, DMSO-d $\left.{ }_{6}\right): \delta=11.08(\mathrm{~s}, 1 \mathrm{H}, \mathrm{NH}), 7.82(d, J=8.3 \mathrm{~Hz}$, $1 \mathrm{H}, 6-\mathrm{H}), 6.85(d, J=8.3 \mathrm{~Hz}, 1 \mathrm{H}, 7-\mathrm{H}) \mathrm{ppm} ;{ }^{13} \mathrm{C} \mathrm{NMR}$ (100 MHz, DMSO-d 6 ): $\delta=182.6(\mathrm{C}-3), 159.2(\mathrm{C}-2)$, 153.2 (C-7a), 138.9 (C-6), 124.5 (C-3a), 122.5 (C-5), 112.2 (C-7), 101.3 (C-4) ppm;

MS (ESI, MeOH): m/z (\%) = $349.9\left(\left[{ }^{79} \mathrm{M}-\mathrm{H}\right]^{-}, 100\right)$; analysis calcd for $\mathrm{C}_{8} \mathrm{H}_{3} \mathrm{BrINO}_{2}$ (351.93): $\mathrm{C} 27.30, \mathrm{H}$ 0.84, N 3.98; found C 27.03, H 1.11, N 3.61.

\section{5-Bromo-6-iodoisatin (16d)}

Column chromatography (silica gel, $n$-hexane/ethyl acetate, 7:3) afforded 16d $(0.22 \mathrm{~g}, 82 \%)$ as an orange solid; $\mathrm{R}_{F}=0.30$ (silica gel, $n$-hexane/ethyl acetate $7: 3) ; \mathrm{mp}=263-265^{\circ} \mathrm{C}$;

IR (ATR): $v=3449 w, 3281 m, 1736 m, 1589 m, 1332 s$, $1238 m, 1152 m, 1076 w, 1046 w, 899 m, 859 m, 821 m$, $719 m, 657 s, 564 \mathrm{~s} \mathrm{~cm}^{-1}$;

UV-vis $(\mathrm{MeOH}): \lambda(\log \varepsilon)=316 \mathrm{~nm}(3.15)$;

${ }^{1} \mathrm{H}$ NMR (500 MHz, DMSO-d $\left.{ }_{6}\right) \delta=11.15(\mathrm{~s}, 1 \mathrm{H}$, $\mathrm{NH}), 7.75$ (s, 1H, 4-H, $7.46(\mathrm{~s}, 1 \mathrm{H}, 7-\mathrm{H}) \mathrm{ppm} ;{ }^{13} \mathrm{C}$ NMR (125 MHz, DMSO-d d $_{6} \delta=182.7$ (C-3), 159.0 (C-2), 149.2 (C-7a), 127.0 (C-4), 123.2 (C-7), 122.0 (C-7), 119.5 (C-3a), 113.7 (C-6) ppm;

MS (ESI, MeOH): m/z $(\%)=349.9\left(\left[{ }^{79} \mathrm{M}-\mathrm{H}\right]^{-}, 85\right)$, 381.7 ([ $\left.\left.{ }^{79} \mathrm{M}-\mathrm{H}+\mathrm{MeOH}\right]^{-}, 12.5\right)$;

analysis calcd for $\mathrm{C}_{8} \mathrm{H}_{3} \mathrm{BrINO}_{2}$ (351.93): C 27.30, H 0.84, N 3.98; found C 27.07, H 1.14, N 3.68.

\section{5-Bromo-7-chloroisatin (16e)}

Compound 16e $(0.23 \mathrm{~g}, 82 \%)$ was obtained as an orange solid; $\mathrm{R}_{F}=0.47$ (silica gel, toluene/ $n$ heptane/ethyl acetate/formic acid, 80:20:10:3);

$\mathrm{mp}=210-213^{\circ} \mathrm{C}$;

IR (KBr): $v=3432 b r, 3102 m, 1744 v s, 1614 s, 1456 s$, $1432 m, 1384 w, 1290 m, 1270 w, 1218 w, 1170 m$, $1112 w, 1072 w, 1036 w \mathrm{~cm}^{-1}$;

UV-vis $(\mathrm{MeOH}): \lambda(\log \varepsilon)=217$ (4.13), 257 (4.13), 305 (3.13) nm;

${ }^{1} \mathrm{H}$ NMR (500 MHz, DMSO-d $\left.{ }_{6}\right): \delta=11.58(\mathrm{~s}, 1 \mathrm{H}$, $\mathrm{NH}), 7.94(d, J=1.9 \mathrm{~Hz}, 1 \mathrm{H}, 4-\mathrm{H}), 7.65(d, J=1.8$ $\mathrm{Hz}, 1 \mathrm{H}, 6-\mathrm{H}) \mathrm{ppm} ;{ }^{13} \mathrm{C}$ NMR (125 MHz, DMSO-d 6 ): $\delta=182.2(\mathrm{C}-3), 159.3$ (C-2), 146.9 (C-7a), 138.5 (C6), 125.6 (C-4), 121.1 (C-3a), 117.4 (C-7), 114.3 (C5) ppm;

MS (ESI, MeOH): m/z (\%) = $259.9\left([\mathrm{M}-\mathrm{H}]^{-}, 100\right)$, $291.8\left([\mathrm{M}-\mathrm{H}+\mathrm{MeOH}]^{-}, 21\right)$;

analysis calcd for $\mathrm{C}_{8} \mathrm{H}_{3} \mathrm{BrClNO}_{2}$ (260.47): C 36.89, H 1.16, N 5.38; found C 36.77, H 1.37, N 5.16.

\section{5-Bromo-7-iodoisatin (16f)}

Compound 16f (0.24 g, 96\%) was obtained as an orange solid; $\mathrm{R}_{F}=0.45$ (silica gel, toluene $/ n$ heptane/ethyl acetate/formic acid, 80:20:10:3);

$\mathrm{mp}=257-260^{\circ} \mathrm{C}$;

IR $(\mathrm{KBr}): v=3422 \mathrm{br}, 3228 \mathrm{br}, 1774 v s, 1750 v s$, $1740 v s, 1608 s, 1458 s, 1448 v s, 1420 m, 1376 m$, $1302 m, 1264 w, 1214 w, 1156 m, 1076 m, 1032 m, 876 m$, $690 \mathrm{~m}, 668 \mathrm{~m}, 544 \mathrm{~m} \mathrm{~cm}^{-1}$;

UV-vis (MeOH): $\lambda(\log \varepsilon)=203$ (4.29), 288 (4.29), 359 (4.29), 313 (3.29) nm;

${ }^{1} \mathrm{H}$ NMR (400 MHz, DMSO-d $\left.\mathrm{d}_{6}\right): \delta=11.12(s, 1 \mathrm{H}$, $\mathrm{NH}), 8.13(d, J=1.9 \mathrm{~Hz}, 1 \mathrm{H}, 6-\mathrm{H}), 7.66(d, J=1.7$ $\mathrm{Hz}, 1 \mathrm{H}, 4-\mathrm{H}) \mathrm{ppm} ;{ }^{13} \mathrm{C}$ NMR (100 MHz, DMSO-d 6 ): $\delta=183.2(\mathrm{C}-3), 159.5$ (C-2), 152.0 (C-7a), 146.7 (C6), 126.2 (C-4), 120.7 (C-3a), 114.9 (C-5), 79.9 (C-7) ppm;

MS (ESI, $\mathrm{MeOH}): \mathrm{m} / \mathrm{z}(\%)=349.9\left([\mathrm{M}-\mathrm{H}]^{-}, 100\right)$, 382.4 ([M-H+MeOH] $]^{-}$, 29); analysis calcd for $\mathrm{C}_{8} \mathrm{H}_{3} \mathrm{BrINO}_{2}$ (351.93): C 27.30, H 0.84, N 3.98; found C 27.11, H 1.07, N 3.78. 


\section{5-Bromo-6-fluoroisatin (16g)}

Compound 16g $(0.236 \mathrm{~g}, 75 \%)$ was obtained as an orange solid; $\mathrm{R}_{F}=0.35$ (silica gel, toluene $/ n$ heptane/ethyl acetate/formic acid, 80:20:10:3); $\mathrm{mp}=239-242^{\circ} \mathrm{C}$;

IR $(\mathrm{KBr}): v=3448 b r, 3196 m, 3104 w, 3066 w, 1766 s$, $1750 \mathrm{vs}, 1736 \mathrm{~s}, 1718 \mathrm{~s}, 1624 \mathrm{vs}, 1478 \mathrm{~m}, 1442 \mathrm{~m}$, $1380 w, 1328 s, 1278 m, 1234 m, 1212 w, 1172 s, 1090 w$, $1010 m, 904 m, 886 m, 746 m, 676 m, 660 \mathrm{~m} \mathrm{~cm}^{-1}$;

UV-vis $(\mathrm{MeOH}): \lambda(\log \varepsilon)=212$ (4.10), 251 (4.10), 406 (3.10) nm;

${ }^{1} \mathrm{H}$ NMR (500 MHz, DMSO-d $\left.\mathrm{d}_{6}\right): \delta=11.28(\mathrm{~s}, 1 \mathrm{H}$, $\mathrm{NH}), 7.87(d, J=7.0 \mathrm{~Hz}, 1 \mathrm{H}, 4-\mathrm{H}), 6.92(\mathrm{~d}, J=8.9$ $\mathrm{Hz}, 1 \mathrm{H}, 7-\mathrm{H}) \mathrm{ppm} ;{ }^{13} \mathrm{C}$ NMR (125 MHz, DMSO-d 6 ): $\delta=181.9(d, J=1.2 \mathrm{~Hz}, \mathrm{C}-3), 163.9(d, J=255.0 \mathrm{~Hz}$, C-6), 159.7 (s, C-2), 152.5 ( $d, J=13.0 \mathrm{~Hz}, \mathrm{C}-7 \mathrm{a})$, $130.3(d, J=3.1 \mathrm{~Hz}, \mathrm{C}-4), 116.4(d, J=3.0 \mathrm{~Hz}, \mathrm{C}-3 \mathrm{a})$, $110.1(d, J=23.5 \mathrm{~Hz}, \mathrm{C}-5), 102.1(d, J=28.3 \mathrm{~Hz}$, C-7) ppm; ${ }^{19} \mathrm{~F}$ NMR (470 MHz, DMSO-d 6 ): $\delta=$ $-91.66(d d, J=8.8,6.9 \mathrm{~Hz}) \mathrm{ppm}$;

MS (ESI, MeOH): m/z (\%) = $242\left(\left[{ }^{79} \mathrm{M}-\mathrm{H}\right]^{-}\right), 274$ $\left(\left[{ }^{79} \mathrm{M}-\mathrm{H}+\mathrm{MeOH}\right]^{-}\right)$;

analysis calcd for $\mathrm{C}_{8} \mathrm{H}_{3} \mathrm{BrFNO}_{2}$ (244.02): C 39.38, $\mathrm{H}$ 1.24, N 5.74; found C 39.16, H 1.45, N 5.55.

\section{5-Bromo-7-fluoroisatin (16h)}

Column chromatography (silica gel, $n$-hexane/ethyl acetate, 7:3) gave compound $\mathbf{1 6 h}(0.012 \mathrm{~g}, 4 \%)$ as an amorphous brown solid; $\mathrm{R}_{F}=0.37$ (silica gel, $n$ hexane/ethyl acetate, $7: 3) ; \mathrm{mp}=219-222^{\circ} \mathrm{C}$;

${ }^{1} \mathrm{H}$ NMR (500 MHz, DMSO-d 6 ): $\delta=11.66(\mathrm{~s}, 1 \mathrm{H}$, $\mathrm{NH}), 7.87(d d, J=9.7,1.7 \mathrm{~Hz}, 1 \mathrm{H}, 6-\mathrm{H}), 7.56(d, J=$ $1.4 \mathrm{~Hz}, 1 \mathrm{H}, 4-\mathrm{H}) \mathrm{ppm} ;{ }^{13} \mathrm{C}$ NMR (125 MHz, DMSO$\left.\mathrm{d}_{6}\right) \delta=181.9(s, \mathrm{C}-3), 158.8(s, \mathrm{C}-2), 147.2(d, J=$ $249.9 \mathrm{~Hz}, \mathrm{C}-7), 136.8(d, J=13.3 \mathrm{~Hz}, \mathrm{C}-7 \mathrm{a}), 126.9$ $(d, J=20.7 \mathrm{~Hz}, \mathrm{C}-6), 123.1(d, J=3.6 \mathrm{~Hz}, \mathrm{C}-4), 121.6$ $(d, J=4.3 \mathrm{~Hz}, \mathrm{C}-3 \mathrm{a}), 113.5(d, J=6.8 \mathrm{~Hz}, \mathrm{C}-5) \mathrm{ppm}$; ${ }^{19} \mathrm{~F}$ NMR $\left(470 \mathrm{MHz}, \mathrm{DMSO}-\mathrm{d}_{6}\right): \delta=-130.60(d, J=$ $9.7 \mathrm{~Hz}, \mathrm{~F}) \mathrm{ppm}$;

MS (ESI, MeOH): m/z (\%) = $242\left(\left[{ }^{79} \mathrm{M}-\mathrm{H}\right]^{-}\right)$;

analysis calcd for $\mathrm{C}_{8} \mathrm{H}_{3} \mathrm{BrFNO}_{2}$ (244.02): C 39.38, H 1.24, N 5.74; found 39.11, H 1.47, N 5.63.

\section{5,7-Dibromo-4-chloroisatin (17a)}

Column chromatography (silica gel, $n$-hexane/ethyl acetate, $7: 4)$ gave $17 \mathbf{a}(0.018 \mathrm{~g}, 5 \%)$ as a red solid; $\mathrm{R}_{F}$ $=0.51$ (silica gel, $n$-hexane/ethyl acetate, $7: 4)$;

$\mathrm{mp}=240-244^{\circ} \mathrm{C}$;

${ }^{1} \mathrm{H}$ NMR (400 MHz, DMSO-d 6 ): $\delta=11.56(s, 1 \mathrm{H}$, $\mathrm{NH}), 8.23(s, 1 \mathrm{H}, 6-\mathrm{H}) \mathrm{ppm} ;{ }^{13} \mathrm{C}$ NMR $(100 \mathrm{MHz}$, DMSO-d $\left.\mathrm{d}_{6}\right): \delta=213.8(\mathrm{C}-3), 158.7(\mathrm{C}-2), 154.6(\mathrm{C}-$ 7a), 149.9 (C-4), 142.2 (C-6), 130.1 (C-3a), 115.9 (C5), 103.8 (C-7) ppm; MS (ESI, MeOH): m/z (\%) = $361.8 \quad\left(\left[{ }^{35 / 79 / 81} \mathrm{M}+\mathrm{H}\right]^{+}, \quad 26\right), \quad 391.9$ $\left(\left[{ }^{35 / 79 / 79} \mathrm{M}+\mathrm{Na}+\mathrm{MeOH}\right]^{+}, \quad 55\right), 335.9\left(\left[{ }^{35 / 79 / 79} \mathrm{M}-\mathrm{H}\right]^{-}\right.$, 48), 367.7 ([ $\left.\left.{ }^{35 / 79 / 79} \mathrm{M}-\mathrm{H}+\mathrm{MeOH}\right]^{-}, 14\right)$;

analysis calcd for $\mathrm{C}_{8} \mathrm{H}_{2} \mathrm{Br}_{2} \mathrm{ClNO}_{2}$ (339.37): C 28.31, H 0.59, N 4.13; found C 28.03, H 0.86, N 4.97

\section{5,7-Dibromo-6-chloroisatin (17b)}

Column chromatography (silica gel, $n$-hexane/ethyl acetate, 7:4) gave $\mathbf{1 7 b}(0.010 \mathrm{~g}, 3 \%)$ as a slightly orange solid; $\mathrm{R}_{F}=0.59$ (silica gel, $n$-hexane/ethyl acetate, $7: 4) ; \mathrm{mp}=188-193^{\circ} \mathrm{C}$;
${ }^{1} \mathrm{H}$ NMR (500 MHz, DMSO-d 6 ): $\delta=11.55$ (s, 1H, NH), 7.90 (s, 1H, 4-H) ppm;

${ }^{13} \mathrm{C}$ NMR (125 MHz, DMSO-d 6 ): $\delta=181.9(\mathrm{C}-3)$, 159.6 (C-2), 150.0 (C-7a), 141.0 (C-6), 127.4 (C-4), 119.2 (C-3a), 115.0 (C-5), 106.5 (C-7) ppm;

MS (ESI, MeOH): m/z $(\%)=335.9\left(\left[\left[^{35 / 79 / 79} \mathrm{M}-\mathrm{H}\right]^{-}\right.\right.$, 54), 367.7 ([ $\left.\left[{ }^{35 / 79 / 79} \mathrm{M}-\mathrm{H}+\mathrm{MeOH}\right]^{-}, 8\right)$;

analysis calcd for $\mathrm{C}_{8} \mathrm{H}_{2} \mathrm{Br}_{2} \mathrm{ClNO}_{2}$ (339.37): C 28.31, H 0.59, N 4.13; found C 28.04, H 0.76, N 4.00.

\section{5,7-Dibromo-4-iodoisatin (17c)}

Column chromatography (silica gel, $n$-hexane/ethyl acetate, $7: 4)$ gave $17 \mathrm{c}(0.03 \mathrm{~g}, 8 \%)$ as a red solid; $\mathrm{R}_{F}$ $=0.63$ (silica gel, $n$-hexane/ethyl acetate 7:4); $\mathrm{mp}=234-238^{\circ} \mathrm{C}$;

${ }^{1} \mathrm{H}$ NMR (400 MHz, DMSO-d $\left.\mathrm{d}_{6}\right): \delta=11.36(s, 1 \mathrm{H}$, $\mathrm{NH}), 8.12(s, 1 \mathrm{H}, 6-\mathrm{H}) \mathrm{ppm} ;{ }^{13} \mathrm{C} \mathrm{NMR}(100 \mathrm{MHz}$, DMSO-d $\left.{ }_{6}\right): \delta=181.5(\mathrm{C}-3), 158.6(\mathrm{C}-2), 150.3(\mathrm{C}-$ 7a), 140.6 (C-6), 124.5 (C-3a), 123.5 (C-5), 105.4 (C4), 100.0 (C-7) ppm;

MS (ESI, MeOH): m/z $(\%)=427.9\left(\left[\left[^{79 / 79} \mathrm{M}-\mathrm{H}\right]^{-}, 44\right)\right.$, 459.6 ([ $\left.\left.{ }^{79 / 79} \mathrm{M}-\mathrm{MeOH}\right]^{-}, 25\right)$;

analysis calcd for $\mathrm{C}_{8} \mathrm{H}_{2} \mathrm{Br}_{2} \mathrm{INO}_{2}$ (430.82): C 22.30, $\mathrm{H}$ 0.47, N 3.25; found C 22.13, H 0.69, N 3.03.

\section{5,7-Dibromo-6-iodoisatin (17d)}

Column chromatography (silica gel, $n$-hexane/ethyl acetate, 7:3) afforded compound $\mathbf{1 7 d}(0.011 \mathrm{~g}, 3 \%)$ as a lightly orange solid; $\mathrm{R}_{F}=0.43$ (silica gel,

$n$-hexane/ethyl acetate $7: 3) ; \operatorname{mp} 225-228^{\circ} \mathrm{C}$;

${ }^{1} \mathrm{H}$ NMR (500 MHz, DMSO-d 6 ): $\delta=11.37$ (s, 1H, $\mathrm{NH}), 7.78$ (s, 1H, 4-H) ppm; ${ }^{13} \mathrm{C}$ NMR $(125 \mathrm{MHz}$, DMSO-d 6 ): $\delta=182.6$ (C-3), 159.7 (C-2), 147.7

(C-7a), 125.3 (C-4), 123.0 (C-5), 122.2 (C-3a), 120.7 (C-7), 114.0 (C-6) ppm;

MS (ESI, MeOH): m/z $(\%)=427.9\left([\mathrm{M}-\mathrm{H}]^{-}, 50\right)$, 459.7 ([M-H+MeOH]$\left.]^{-}, 18\right)$;

analysis calcd for $\mathrm{C}_{8} \mathrm{H}_{2} \mathrm{Br} 2 \mathrm{INO} 2$ (430.82): $\mathrm{C} 22.31, \mathrm{H}$ 0.47 , N 3.25; found: 21.98, H 0.74, N 3.02.

\subsubsection{General procedure $D$}

To a solution of the isatin (1 eq.) in $\mathrm{EtOH}$ at $35^{\circ} \mathrm{C}$ a solution of hydroxylammonium chloride (1.4 eq.) in water $(1 \mathrm{~mL})$ was added. The solution was heated to $80^{\circ} \mathrm{C}$ until the reaction was completed (as indicated by TLC) 7 . Upon cooling (ice) the product precipitated; it was filtered off, washed with water and dried.

\section{4-Chloroisatin-3Z-oxime (18)}

Compound 18a $(0.44 \mathrm{~g}, 81 \%)$ was obtained as a yellow solid; $\mathrm{R}_{F}=0.25$ (silica gel, toluene $/ n$ heptane/ethyl acetate/formic acid, 80:20:10:3);

$\mathrm{mp}=231-233^{\circ} \mathrm{C}$;

IR (KBr): $v=3244 s, 3194 s, 3082 m, 3002 m, 2936 m$, $2852 w, 1702 v s, 1654 w, 1622 m, 1600 s, 1588 s, 1484 m$, $1444 \mathrm{~s}, 1426 \mathrm{~m}, 1404 \mathrm{~m}, 1318 \mathrm{~m}, 1292 \mathrm{~m}, 1250 \mathrm{~m}$, $1224 w, 1172 s, 1146 m, 1072 m, 1044 m, 1036 m, 972 m$, $940 m, 770 s, 734 m, 722 \mathrm{~m} \mathrm{~cm}^{-1}$;

UV-vis $(\mathrm{MeOH}): \lambda(\log \varepsilon)=210$ (4.19), 228 (4.19), $254 \mathrm{~nm}$ (3.49), 260 (4.19), 300 (3.49) nm;

${ }^{1} \mathrm{H}$ NMR (400 MHz, DMSO-d 6 ): $\delta=13.47(s, 1 \mathrm{H}$, $\mathrm{NOH}), 10.90(s, 1 \mathrm{H}, \mathrm{NH}), 7.29(t, J=8.0 \mathrm{~Hz}, 1 \mathrm{H}, 6-$ $\mathrm{H}), 7.04(d, J=8.2 \mathrm{~Hz}, 1 \mathrm{H}, 5-\mathrm{H}), 6.81(d, J=7.8 \mathrm{~Hz}$, 1H, 7-H) ppm; ${ }^{13} \mathrm{C}$ NMR (125 MHz, DMSO-d 6 ): 
$\delta=157.2(\mathrm{C}-2), 143.2(\mathrm{C}-3), 142.3$ (C-7a), 131.6 (C-6), 127.1 (C-4), 122.9 (C-5), 116.5 (C-3a), 108.8 (C-7) ppm;

MS (ESI, $\mathrm{MeOH}): \mathrm{m} / \mathrm{z}(\%)=197.1\left([\mathrm{M}+\mathrm{H}]^{+}, 26\right)$, $219.0\left([\mathrm{M}+\mathrm{Na}]^{+}, 100\right), 314.9\left([3 \mathrm{M}+\mathrm{K}+\mathrm{H}]^{2+}, 32\right)$, $414.8\left([2 \mathrm{M}+\mathrm{Na}]^{+}, 47\right), 195.0\left([\mathrm{M}-\mathrm{H}]^{-}, 100\right), 230.8$ $\left(\left[\mathrm{M}+{ }^{35} \mathrm{Cl}\right]^{-}, 7\right), 390.6\left([2 \mathrm{M}-\mathrm{H}]^{-}, 15\right)$;

analysis calcd for $\mathrm{C}_{8} \mathrm{H}_{5} \mathrm{ClN}_{2} \mathrm{O}_{2}$ (196.59): $\mathrm{C} 48.88, \mathrm{H}$ 2.56, N 14.25; found C 48.66, H 2.75, N 13.99.

\section{6-Chloroisatin-3Z-oxime (18b)}

Compound 18b $(0.42 \mathrm{~g}, 66 \%)$ was obtained as a yellow solid; $\mathrm{R}_{F}=0.14$ (silica gel, toluene $/ n$ heptane/ethyl acetate/formic acid, 80:20:10:3); $\mathrm{mp}=246-250^{\circ} \mathrm{C}$;

IR (KBr): $v=3422 m, 3210 s, 3178 s, 2908 m, 1736 v s$, $1716 s, 1622 s, 1508 w, 1480 w, 1440 m, 1370 w, 1340 m$, $1286 w, 1246 w, 1218 w, 1186 w, 1112 w, 1076 m, 1020 s$, $818 m, 666 \mathrm{~m} \mathrm{~cm}^{-1}$;

UV-vis $(\mathrm{MeOH}): \lambda(\log \varepsilon)=259$ (4.31), 296 (4.01), 365 (3.31) nm; ${ }^{1} \mathrm{H}$ NMR (500 MHz, DMSO-d 6 ): $\delta=$ $13.45(s, 1 \mathrm{H}, \mathrm{NOH}), 10.83(s, 1 \mathrm{H}, \mathrm{NH}), 7.92(d, J=$ $8.1 \mathrm{~Hz}, 1 \mathrm{H}, 4-\mathrm{H}), 7.06(d d, J=8.1,1.9 \mathrm{~Hz}, 1 \mathrm{H}, 5-\mathrm{H})$, $6.89(d, J=1.9 \mathrm{~Hz}, 1 \mathrm{H}, 7-\mathrm{H}) \mathrm{ppm} ;{ }^{13} \mathrm{C} \mathrm{NMR}(125$ MHz, DMSO-d $\left.\mathrm{d}_{6}\right): \delta=164.3(\mathrm{C}-2), 143.9(\mathrm{C}-3), 143.3$ (C-7a), 136.1 (C-6), 128.2 (C-4), 121.8 (C-5), 114.7 (C-3a), 110.3 (C-7) ppm;

MS (ESI, MeOH): m/z $(\%)=197.1\left([\mathrm{M}+\mathrm{H}]^{+}, 100\right)$, $219.1\left([\mathrm{M}+\mathrm{Na}]^{+}, 94\right), 314.1\left([3 \mathrm{M}+\mathrm{H}+\mathrm{K}]^{2+}, 13\right), 329.7$ $\left([3 \mathrm{M}+\mathrm{H}+\mathrm{K}+\mathrm{MeOH}]^{2+}, 19\right), 393.1\left([2 \mathrm{M}+\mathrm{H}]^{+}, 13\right)$, $415.9\left([2 \mathrm{M}+\mathrm{Na}]^{+}, 34\right), 195.0\left([\mathrm{M}-\mathrm{H}]^{-}, 100\right), 412.9$ $\left([2 \mathrm{M}-2 \mathrm{H}+\mathrm{Na}]^{-}, 3\right)$;

analysis calcd for $\mathrm{C}_{8} \mathrm{H}_{5} \mathrm{ClN}_{2} \mathrm{O}_{2}$ (196.59): $\mathrm{C} 48.88, \mathrm{H}$ 2.56, N 14.25; found C 48.59, H 2.72, N 13.94.

\section{4-Iodoisatin-3Z-oxime (18c)}

Compound 18c $(0.16 \mathrm{~g}, 71 \%)$ was obtained as a yellow solid; $\mathrm{R}_{F}=0.26$ (silica gel, toluene $/ n$ heptane/ethyl acetate/formic acid, 80:20:10:3); $\mathrm{mp}=234-236^{\circ} \mathrm{C}$;

IR (KBr): $v=3266 s, 3168 s, 3128 s, 3074 s, 3048 m$, $3014 m, 2900 m, 2838 m, 2742 m, 2690 m, 2564 w$, $1704 v s, 1620 m, 1596 s, 1574 s, 1482 m, 1436 s, 1404 m$, $1318 s, 1288 m, 1258 s, 1220 m, 1172 s, 1128 m, 1114 m$, $1078 m, 1040 s, 960 s, 916 m, 764 s, 724 m, 702 s, 656 m$, $638 \mathrm{~m} \mathrm{~cm}^{-1}$;

UV-vis $(\mathrm{MeOH}): \lambda(\log \varepsilon)=204$ (4.12), 237 (4.12), 258 (4.12), 313 (3.42) nm;

${ }^{1} \mathrm{H}$ NMR (400 MHz, DMSO-d 6$): \delta=13.48(s, 1 \mathrm{H}$, $\mathrm{NOH}), 10.78(s, 1 \mathrm{H}, \mathrm{NH}), 7.47(d, J=7.9 \mathrm{~Hz}, 1 \mathrm{H}, 5-$ $\mathrm{H}), 7.02(t, J=7.9 \mathrm{~Hz}, 1 \mathrm{H}, 6-\mathrm{H}), 6.87(d, J=7.7 \mathrm{~Hz}$, $1 \mathrm{H}, 7-\mathrm{H}) \mathrm{ppm} ;{ }^{13} \mathrm{C}$ NMR (100 MHz, DMSO-d 6 ): $\delta=$ 157.6 (C-2), 143.2 (C-3), 142.4 (C-8), 132.8 (C-6), 131.6 (C-5), 121.4 (C-3a), 109.7 (C-7), 87.0 (C-4) ppm;

MS (ESI, MeOH): m/z $(\%)=289.1\left([\mathrm{M}+\mathrm{H}]^{+}, 86\right)$, $311.0\left([\mathrm{M}+\mathrm{Na}]^{+}, 93\right), 342.5\left([\mathrm{M}+\mathrm{Na}+\mathrm{MeOH}]^{+}, 28\right)$, $451.9\left([3 \mathrm{M}+\mathrm{K}+\mathrm{H}]^{+}, 43\right), 598.6\left([2 \mathrm{M}+\mathrm{Na}]^{+}, 100\right)$, 287.1 ([M-H] $\left.]^{-}, 100\right)$;

analysis calcd for $\mathrm{C}_{8} \mathrm{H}_{5} \mathrm{IN}_{2} \mathrm{O}_{2}$ (288.04): C 33.36, $\mathrm{H}$ 1.75, N 9.73; found C 33.11, H 1.92, N 9.53.

\section{6-Iodoisatin-3Z-oxime (18d)}

Compound 18d $(0.10 \mathrm{~g}, 57 \%)$ was obtained as a yellow solid; $\mathrm{R}_{F}=0.15$ (silica gel, toluene/ $n$ heptane/ethyl acetate/formic acid, 80:20:10:3); $\mathrm{mp}=260-262^{\circ} \mathrm{C}$;

IR (KBr): $v=3420 m, 3240 s, 2906 w, 1726 v s, 1682 m$, $1664 \mathrm{~m}, 1610 \mathrm{vs}, 1468 \mathrm{~m}, 1428 \mathrm{~m}, 1352 \mathrm{~m}, 1318 \mathrm{~m}$, $1286 m, 1246 w, 1186 w, 1112 m, 1030 s, 894 m, 860 m$, $816 m, 758 m, 730 m, 680 m, 658 m \mathrm{~cm}^{-1}$;

UV-vis $(\mathrm{MeOH}): \lambda(\log \varepsilon)=262$ (4.17), 304 (4.17) 366 (3.47) nm;

${ }^{1} \mathrm{H}$ NMR (400 MHz, DMSO-d 6 ): $\delta=13.44(s, 1 \mathrm{H}$, $\mathrm{NOH}), 10.75(s, 1 \mathrm{H}, \mathrm{NH}), 7.69(d, J=7.9 \mathrm{~Hz}, 1 \mathrm{H}, 5-$ $\mathrm{H}), 7.40(d, J=7.9 \mathrm{~Hz}, 1 \mathrm{H}, 4-\mathrm{H}), 7.21(s, 1 \mathrm{H}, 7-\mathrm{H})$ ppm; ${ }^{13} \mathrm{C}$ NMR (125 MHz, DMSO-d 6 ): $\delta=164.1$ (C2), 143.7 (C-3), 143.6 (C-7a), 130.8 (C-5), 128.3 (C4), 118.6 (C-7), 115.3 (C-3a), 98.6 (C-6) ppm;

MS (ESI, MeOH): m/z $(\%)=289.1\left([\mathrm{M}+\mathrm{H}]^{+}, 100\right)$, $311.0\left([\mathrm{M}+\mathrm{Na}]^{+}, 85\right), 576.7\left([2 \mathrm{M}+\mathrm{H}]^{+}, 23\right), 596.5$ $\left([4 \mathrm{M}+\mathrm{K}+\mathrm{H}]^{2+}, 37\right), 598.6\left([2 \mathrm{M}+\mathrm{Na}]^{+}, 57\right), 287.1([\mathrm{M}-$ $\left.\mathrm{H}]^{-}, 100\right), 596.6$ ([2M-2H+Na] $\left.]^{-}, 4\right)$;

analysis calcd for $\mathrm{C}_{8} \mathrm{H}_{5} \mathrm{IN}_{2} \mathrm{O}_{2}(288.04)$ : C $33.36, \mathrm{H}$ 1.75, N 9.73; found C 33.16, H 1.96, N 9.57.

\section{7-Chloroisatin-3Z-oxime (18e)}

Compound 18e $(0.30 \mathrm{~g}, 46 \%)$ was obtained as a yellow solid; $\mathrm{R}_{F}=0.20$ (silica gel, toluene/ $n$ heptane/ethyl acetate/formic acid, 80:20:10:3); $\mathrm{mp}=255-260^{\circ} \mathrm{C}$ (lit.: $\left.{ }^{34} 300^{\circ} \mathrm{C}\right)$;

MS (ESI, MeOH): m/z $(\%)=197.1\left([\mathrm{M}+\mathrm{H}]^{+}, 16\right)$, $213.9\left(\left[\mathrm{M}+\mathrm{NH}_{4}\right]^{+}, 15\right), 219.0\left([\mathrm{M}+\mathrm{Na}]^{+}, 30\right), 250.6$ $\left([\mathrm{M}+\mathrm{Na}+\mathrm{MeOH}]^{+}, 8\right), 392.8\left([2 \mathrm{M}+\mathrm{H}]^{+}, 16\right), 414.8$ $\left([2 \mathrm{M}+\mathrm{Na}]^{+}, 100\right), 416.8\left(\left[\mathrm{M}+{ }^{37} \mathrm{M}+\mathrm{Na}\right]^{+}, 66\right), 195.0$ $\left(\left[{ }^{35} \mathrm{M}-\mathrm{H}\right]^{-}, 100\right)$.

\section{7-Iodoisatin-3Z-oxime (18f)}

Compound 18f (0.39 g, 94\%) was obtained as a yellow solid; $\mathrm{R}_{F}=0.21$ (silica gel, toluene/ $n$ heptane/ethyl acetate/formic acid, 80:20:10:3); $\mathrm{mp}=270-272^{\circ} \mathrm{C}$;

IR (KBr): $v=3314 m, 3188 s, 3154 s, 3074 m, 2926 m$, $2882 m, 2782 m, 2658 w, 2360 w, 2030 w, 1748 v s$, $1634 m, 1604 s, 1572 m, 1454 m, 1420 s, 1386 w, 1336 s$, $1290 w, 1218 m, 1172 m, 1124 m, 1060 w, 1020 v s$, $790 \mathrm{~m}, 730 \mathrm{~m}, 682 \mathrm{~s} \mathrm{~cm}^{-1}$;

UV-vis $(\mathrm{MeOH}): \lambda(\log \varepsilon)=228$ (3.54), 235 (4.24), 254 (4.24), 260 (4.24), 304 (4.24), 372 (3.54) nm; ${ }^{1} \mathrm{H}$ NMR (400 MHz, DMSO-d 6$): \delta=13.51(s, 1 \mathrm{H}, \mathrm{NOH})$, $10.67(s, 1 \mathrm{H}, \mathrm{NH}), 7.96(d, J=7.3 \mathrm{~Hz}, 1 \mathrm{H}, 4-\mathrm{H}), 7.71$ $(d, J=8.0 \mathrm{~Hz}, 1 \mathrm{H}, 6-\mathrm{H}), 6.83(t, J=7.7 \mathrm{~Hz}, 1 \mathrm{H}, 5-\mathrm{H})$ ppm; ${ }^{13} \mathrm{C}$ NMR (125 MHz, DMSO-d 6 ): $\delta=164.7$ (C2), 145.7 (C-3), 144.7 (C-7a), 141.1 (C-6), 126.8 (C4), 124.5 (C-5), 117.2 (C-3a), 76.2 (C-7) ppm;

MS (ESI, $\mathrm{MeOH}): \mathrm{m} / \mathrm{z}(\%)=289.1\left([\mathrm{M}+\mathrm{H}]^{+}, 84\right)$, $305.8\left(\left[\mathrm{M}+\mathrm{NH}_{4}\right]^{+}, 95\right), 311.0\left([\mathrm{M}+\mathrm{Na}]^{+}, 100\right), 326.7$ $\left([\mathrm{M}+\mathrm{K}]^{+}, 54\right), 342.5\left([\mathrm{M}+\mathrm{Na}+\mathrm{MeOH}]^{+}, 39\right), 287.0$ ([M-H]- 100);

analysis calcd for $\mathrm{C}_{8} \mathrm{H}_{5} \mathrm{IN}_{2} \mathrm{O}_{2}$ (288.04): C 33.36, H 1.75, N 9.73; found C 33.07, H 1.96, N 9.48.

\section{6-Fluoroisatin-3Z-oxime (18g)}

Compound 18g (0.17 g, 75\%) was obtained as a yellow solid; $\mathrm{R}_{F}=0.15$ (silica gel, toluene/ $n$ heptane/ethyl acetate/formic acid, 80:20:10:3); 
$\mathrm{mp}=255-257^{\circ} \mathrm{C}$;

IR $(\mathrm{KBr}): v=3420 m, 3174 s, 2920 m, 1736 v s, 1666 m$, $1628 v s, 1606 m, 1496 m, 1448 s, 1372 s, 1344 m, 1328 m$, $1300 m, 1268 w, 1250 w, 1234 w, 1186 m, 1134 s, 1096 s$, $1022 v s, 854 m, 816 m, 786 m, 768 m, 744 m, 716 m, 668 s$ $\mathrm{cm}^{-1}$;

UV-vis $(\mathrm{MeOH}): \lambda(\log \varepsilon)=255$ (4.19), 291 (3.89), $258(3.19) \mathrm{nm}$;

${ }^{1} \mathrm{H}$ NMR $\left(500 \mathrm{MHz}, \mathrm{DMSO}-\mathrm{d}_{6}\right): \delta=13.29(s, 1 \mathrm{H}$, $\mathrm{NOH}), 10.83(s, 1 \mathrm{H}, \mathrm{NH}), 7.96(d d, J=8.4,5.9 \mathrm{~Hz}$, $1 \mathrm{H}, 4-\mathrm{H}), 6.80$ (ddd, $J=10.0,8.4,2.4 \mathrm{~Hz}, 1 \mathrm{H}, 5-\mathrm{H})$, $6.69(d d, J=9.2,2.4 \mathrm{~Hz}, 1 \mathrm{H}, 7-\mathrm{H}) \mathrm{ppm} ;{ }^{13} \mathrm{C} \mathrm{NMR}$ $\left(125 \mathrm{MHz}\right.$, DMSO-d $\left.{ }_{6}\right): \delta=164.7(\mathrm{C}-2), 164.0$ $(d, J=248.5 \mathrm{~Hz}, \mathrm{C}-6), 144.8(d, J=12.8 \mathrm{~Hz}, \mathrm{C}-7 \mathrm{a})$, $143.2(d, J=2.3 \mathrm{~Hz}, \mathrm{C}-3), 129.0(d, J=10.3 \mathrm{~Hz}, \mathrm{C}-$ 4), $112.6(d, J=2.8 \mathrm{~Hz}, \mathrm{C}-3 \mathrm{a}), 108.4(d, J=22.7 \mathrm{~Hz}$, C-5), $98.5(d, J=27.3 \mathrm{~Hz}, \mathrm{C}-7) \mathrm{ppm} ;{ }^{19} \mathrm{~F}$ NMR (470 MHz, DMSO-d 6 F: $\delta=-105.69(t d, J=9.6,5.9 \mathrm{~Hz})$ ppm;

MS (ESI, MeOH): m/z $(\%)=181.1\left([\mathrm{M}+\mathrm{H}]^{+}, 100\right)$, $197.9\left(\left[\mathrm{M}+\mathrm{NH}_{4}\right]^{+}, 12\right), 203.0\left([\mathrm{M}+\mathrm{Na}]^{+}, 50\right), 234.5$ $\left([\mathrm{M}+\mathrm{Na}+\mathrm{MeOH}]^{+}, 10\right), 243.7\left([\mathrm{M}+\mathrm{Na}+\mathrm{MeCN}]^{+}, 44\right)$, $360.9\left([2 \mathrm{M}+\mathrm{H}]^{+}, 95\right), 382.9\left([2 \mathrm{M}+\mathrm{Na}]^{+}, 95\right), 179.1$ $\left([\mathrm{M}-\mathrm{H}]^{-}, 100\right), 236.8\left(\left[\mathrm{M}-\mathrm{H}+\mathrm{Na}^{35} \mathrm{Cl}\right]-, 20\right), 246.9$ $\left(\left[\mathrm{M}+{ }^{35} \mathrm{Cl}+\mathrm{MeOH}\right]^{-}, 20\right)$;

analysis calcd for $\mathrm{C}_{8} \mathrm{H}_{5} \mathrm{FN}_{2} \mathrm{O}_{2}$ (180.14): C 53.34, H 2.80, N 15.55; found C 53.02, H 3.05, N 15.31 .

\section{7-Fluoroisatin-3Z-oxime (18h)}

Compound $\mathbf{1 8 h}(0.41 \mathrm{~g}, 74 \%)$ was obtained as a yellow solid; $\mathrm{R}_{F}=0.17$ (silica gel, toluene $/ n$ heptane/ethyl acetate/formic acid, 80:20:10:3);

$\mathrm{mp}=224-227^{\circ} \mathrm{C}$;

IR (KBr): $v=3566 m, 3420 m, 3374 m, 3210 s, 3208 s$, $3096 s, 2870 s, 2646 m, 1724 v s, 1682 m, 1644 s, 1598 m$, $1496 s, 1448 s, 1338 s, 294 m, 1260 s, 1208 s, 1052 m$, $1022 s, 946 s, 796 m, 726 m, 714 m, 678 s, 588 m \mathrm{~cm}^{-1}$; UV-vis $(\mathrm{MeOH}): \lambda(\log \varepsilon)=236(4.15), 246$ (4.15), 292 (4.15), 324 (4.15), 333 (4.15) nm;

${ }^{1} \mathrm{H}-\mathrm{NMR}\left(500 \mathrm{MHz}, \mathrm{DMSO}-\mathrm{d}_{6}\right): \delta=13.53(s, 1 \mathrm{H}$, $\mathrm{NOH}), 11.20(s, 1 \mathrm{H}, \mathrm{NH}), 7.79(d, J=7.5 \mathrm{~Hz}, 1 \mathrm{H}, 4-$ $\mathrm{H}), 7.29(d d d, J=10.5,8.5,0.9 \mathrm{~Hz}, 1 \mathrm{H}, 6-\mathrm{H}), 7.03$ $(d d d, J=8.4,7.6,4.7 \mathrm{~Hz}, 1 \mathrm{H}, 5-\mathrm{H}) \mathrm{ppm} ;{ }^{13} \mathrm{C} \mathrm{NMR}$ (125 MHz, DMSO-d $\left.\mathrm{d}_{6}\right): \delta=171.9(\mathrm{C}-2), 164.2(\mathrm{C}-3)$, $146.3(d, J=242.8 \mathrm{~Hz}, \mathrm{C}-7), 143.5(d, J=3.8 \mathrm{~Hz}, \mathrm{C}-$ 4), $129.5(d, J=13.2 \mathrm{~Hz}, \mathrm{C}-7 \mathrm{a}), 123.0(d d, J=15.6$, $4.5 \mathrm{~Hz}, \mathrm{C}-5), 118.9(d, J=17.3 \mathrm{~Hz}, \mathrm{C}-6), 118.4(d, J$ $=4.4 \mathrm{~Hz}, \mathrm{C}-3 \mathrm{a}) \mathrm{ppm} ;{ }^{19} \mathrm{~F}$ NMR (470 MHz, DMSO$\left.\mathrm{d}_{6}\right): \delta=-133.06(d d, J=10.5,4.7 \mathrm{~Hz}) \mathrm{ppm}$;

MS (ESI, MeOH): m/z $(\%)=181.1\left([\mathrm{M}+\mathrm{H}]^{+}, 100\right)$, $198.0\left(\left[\mathrm{M}+\mathrm{NH}_{4}\right]^{+}, 67\right), 203.1\left([\mathrm{M}+\mathrm{Na}]^{+}, 96\right), 219.0$ $\left([\mathrm{M}+\mathrm{K}]^{+}, 13\right), 234.7\left([\mathrm{M}+\mathrm{Na}+\mathrm{MeOH}]^{+}, 13\right), 179.1$ ([M-H]', 100);

analysis calcd for $\mathrm{C}_{8} \mathrm{H}_{5} \mathrm{FN}_{2} \mathrm{O}_{2}$ (180.14): C 53.34, H 2.80, N 15.55; found C 53.00, H 3.13, N 15.28.

\section{6,7-Difluoroisatin-3Z-oxime (18i)}

Compound 18i $(0.11 \mathrm{~g}, 45 \%)$ was obtained as a yellow solid; $\mathrm{R}_{F}=0.18$ (silica gel, toluene $/ n$ heptane/ethyl acetate/formic acid, 80:20:10:3); $\mathrm{mp}=264-266^{\circ} \mathrm{C}$;

IR (KBr): $v=3420 m, 3410 m, 3194 m, 3098 m, 3038 m$, $2926 m, 2826 m, 2660 w, 2548 w, 1744 v s, 1698 m$,
$1652 m, 1628 s, 1524 s, 1446 m, 1420 m, 1358 s, 1296 m$, $1270 m, 1248 m, 1226 m, 1156 m, 1046 s, 1014 s, 936 m$, $820 m, 734 m, 690 m, 626 m \mathrm{~cm}^{-1}$; UV-vis $(\mathrm{MeOH}): \lambda$ $(\log \varepsilon)=248(4.08), 292$ (4.08), $356(3.38) \mathrm{nm}$;

${ }^{1} \mathrm{H}$ NMR $\left(500 \mathrm{MHz}, \mathrm{DMSO}-\mathrm{d}_{6}\right): \delta=13.54(s, 1 \mathrm{H}$, $\mathrm{NOH}), 11.47(s, 1 \mathrm{H}, \mathrm{NH}), 7.78(d d d, J=8.5,4.7,1.3$ $\mathrm{Hz}, 1 \mathrm{H}, 4-\mathrm{H}), 7.01$ (ddd, 11.4, 8.3, 7.2 Hz, 1H, 5-H) ppm; ${ }^{13} \mathrm{C}$ NMR (125 MHz, DMSO-d 6 ): $\delta=164.3$ (C2), $151.8(d d, J=249.1,10.0 \mathrm{~Hz}, \mathrm{C}-6), 142.7(t, J=$ $3.0 \mathrm{~Hz}, \mathrm{C}-3), 135.1$ ( $d d, J=246.5,17.1 \mathrm{~Hz}, \mathrm{C}-7)$, $131.6(d d, J=9.5,4.0 \mathrm{~Hz}, \mathrm{C}-7 \mathrm{a}), 123.7(d d, J=8.5$, $3.7 \mathrm{~Hz}, \mathrm{C}-4), 114.3(t, J=3.0 \mathrm{~Hz}, \mathrm{C}-3 \mathrm{a}), 109.8(d, J=$ $18.7 \mathrm{~Hz}, \mathrm{C}-5) \mathrm{ppm} ;{ }^{19} \mathrm{~F}$ NMR (470 MHz, DMSO$\left.\mathrm{d}_{6}\right) \mathrm{H}: \delta=-132.86\left(d d d, J=21.4,11.5,4.7 \mathrm{~Hz}, \mathrm{~F}_{6}\right)$, $157.46\left(d d d, 3 J=21.5,7.2,1.5 \mathrm{~Hz}, \mathrm{~F}_{7}\right) \mathrm{ppm}$;

MS (ESI, $\mathrm{MeOH}): \mathrm{m} / \mathrm{z}(\%)=199.0\left([\mathrm{M}+\mathrm{H}]^{+}, 16\right)$, $215.9\left(\left[\mathrm{M}+\mathrm{NH}_{4}\right]^{+}, 27\right), 221.0\left([\mathrm{M}+\mathrm{Na}]^{+}, 64\right), 415.9$ $\left([4 \mathrm{M}+\mathrm{K}+\mathrm{H}]^{2+}, 53\right), 418.8\left([2 \mathrm{M}+\mathrm{Na}]^{+}, 100\right), 197.1$ ([M-H] $\left.]^{-}, 100\right), 416.9\left([2 \mathrm{M}-2 \mathrm{H}+\mathrm{Na}]^{-}, 10\right)$;

analysis calcd for $\mathrm{C}_{8} \mathrm{H}_{4} \mathrm{~F}_{2} \mathrm{~N}_{2} \mathrm{O}_{2}$ (166.13): C 57.83, H 2.43, N 16.87; found C 57.61, H 2.68, N 16.64.

\section{4,5-Dimethylisatin-3Z-oxime (18j)}

Compound 18j (0.17 g, 64\%) was afforded as a yellow solid; $\mathrm{R}_{F}=0.11$ (silica gel, toluene $/ n$ heptane/ethyl acetate/formic acid, 80:20:10:3); $\mathrm{mp}=264-266^{\circ} \mathrm{C}$;

IR (KBr): $v=3448 s, 3422 s, 3198 s, 3058 m, 2922 m$, $2878 m, 1700 v s, 1624 s, 1560 w, 1458 m, 1386 w$, $1336 w, 1298 w, 1252 w, 1194 w, 1112 m, 1036 m$, $1016 \mathrm{~m} \mathrm{~cm}^{-1}$;

UV-vis $(\mathrm{MeOH}): \lambda(\log \varepsilon)=254$ (4.09), 259 (4.09), 300 (4.09), 379 (3.39 nm;

${ }^{1} \mathrm{H}$ NMR (500 MHz, DMSO-d 6 ): $\delta=13.10(\mathrm{~s}, 1 \mathrm{H}$, $\mathrm{NOH}), 10.49$ (s, 1H, NH), 7.70 (s, 1H, 6-H), 6.67 (s, 1H, 7-H), 2.20 (s, 3H, 4'-H), 2.15 (s, 3H, 5'-H) ppm; ${ }^{13} \mathrm{C}$ NMR (125 MHz, DMSO-d 6 ): $\delta=165.3$ (C-3), 144.8 (C2), 141.4 (C-7a), 141.3 (C-4), 130.0 (C-5), 128.3 (C-6), 114.3 (C-3a), 111.9 (C-7), 20.7 (C-5'), 19.4 (C-4') ppm;

MS (ESI, $\mathrm{MeOH}): \mathrm{m} / \mathrm{z}(\%)=191.1\left([\mathrm{M}+\mathrm{H}]^{+}, 75\right)$, $213.1\left([\mathrm{M}+\mathrm{Na}]^{+}, 67\right), 305.1\left([3 \mathrm{M}+\mathrm{K}+\mathrm{H}]^{+}, 12\right), 320.7$ $\left([3 \mathrm{M}+\mathrm{K}+\mathrm{H}+\mathrm{MeOH}]^{2+}, 6\right), 380.9\left([2 \mathrm{M}+\mathrm{H}]^{+}, 20\right)$, $400.0\left([4 \mathrm{M}+\mathrm{K}+\mathrm{H}]^{2+}, 25\right), 402.9\left([2 \mathrm{M}+\mathrm{Na}]^{+}, 100\right)$, $189.1\left([\mathrm{M}-\mathrm{H}]^{-}, 100\right), 401.1\left([2 \mathrm{M}+2 \mathrm{H}+\mathrm{Na}]^{-}, 10\right)$; analysis calcd for $\mathrm{C}_{10} \mathrm{H}_{10} \mathrm{~N}_{2} \mathrm{O}_{2}$ (190.20): $\mathrm{C} 63.15, \mathrm{H}$ 5.30, N 14.73; found C 62.87, H 5.56, N 14.47.

\section{5,6-Dimethylisatin-3Z-oxime (18k)}

Compound 18k $(0.16 \mathrm{~g}, 74 \%)$ was obtained as an orange solid; $\mathrm{R}_{F}=0.13$ (silica gel, toluene $/ n$ heptane/ethyl acetate/formic acid, 80:20:10:3);

$\mathrm{mp}=269-272^{\circ} \mathrm{C}$;

IR (KBr): $v=3196 s, 3054 s, 2972 m, 2948 m, 2920 m$, $2880 m, 1702 v s, 1622 s, 1458 s, 1430 m, 1392 m, 1374 m$, $1334 m, 1296 w, 1250 w, 1194 m, 1168 w, 1112 m$, $1034 m, 1014 s, 988 m, 828 m, 816 m, 780 m, 732 m$,

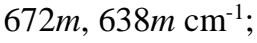

UV-vis $(\mathrm{MeOH}): \lambda(\log \varepsilon)=254$ (4.36), 259 (4.36), 300 (4.36), 383 (3.36) nm;

${ }^{1} \mathrm{H}$ NMR (400 MHz, DMSO-d 6 ): $\delta=13.03(s, 1 \mathrm{H}$, $\mathrm{NOH}), 10.49(s, 1 \mathrm{H}, \mathrm{NH}), 7.72(s, 1 \mathrm{H}, 4-\mathrm{H}), 6.66(s$, $\left.1 \mathrm{H}, 7-\mathrm{H}), 2.22\left(s, 3 \mathrm{H}, 6^{\prime}-\mathrm{H}\right), 2.16\left(s, 3 \mathrm{H}, 5^{\prime}-\mathrm{H}\right)\right) \mathrm{ppm}$; 
${ }^{13} \mathrm{C}$ NMR (100 MHz, DMSO-d 6 ): $\delta=164.7(\mathrm{C}-2)$, 144.3 (C-3), 140.9 (C-7a), 140.8 (C-6), 129.4 (C-5), 127.8 (C-4), 113.8 (C-3a), 111.3 (C-7), 20.2 (C-6'), 18.9 (C-5') ppm;

MS (ESI, $\mathrm{MeOH}): \mathrm{m} / \mathrm{z}(\%)=191.1\left([\mathrm{M}+\mathrm{H}]^{+}, 99\right)$, $213.1\left([\mathrm{M}+\mathrm{Na}]^{+}, 68\right), 305.1\left([3 \mathrm{M}+\mathrm{K}+\mathrm{H}]^{+}, 13\right), 320.7$ $\left([3 \mathrm{M}+\mathrm{K}+\mathrm{H}+\mathrm{MeOH}]^{2+}, 12\right), 380.9\left([2 \mathrm{M}+\mathrm{H}]^{+}, 29\right)$, $400.0\left([4 \mathrm{M}+\mathrm{K}+\mathrm{H}]^{2+}, 44\right), 402.9\left([2 \mathrm{M}+\mathrm{Na}]^{+}, 100\right)$, 189.1 ([M-H $\left.]^{-}, 100\right), 400.9\left([2 \mathrm{M}+2 \mathrm{H}+\mathrm{Na}]^{-}, 8\right)$; analysis calcd for $\mathrm{C}_{10} \mathrm{H}_{10} \mathrm{~N}_{2} \mathrm{O}_{2}$ (190.20): $\mathrm{C} 63.15, \mathrm{H}$ 5.30, N 14.73; found C 62.96, H 5.58, N 14.42.

\section{4-Bromoisatin-3Z-oxime (181)}

Compound 18l $(0.28 \mathrm{~g}, 85 \%)$ was obtained as a yellow solid; $\mathrm{R}_{F}=0.23$ (silica gel, toluene $/ n$ heptane/ethyl acetate/formic acid, 80:20:10:3); $\mathrm{mp}=$ 230-232 ${ }^{\circ} \mathrm{C}$ (lit.: ${ }^{35} 234-236^{\circ} \mathrm{C}$ );

MS (ESI, $\mathrm{MeOH}): \mathrm{m} / \mathrm{z}(\%)=241.1\left([\mathrm{M}+\mathrm{H}]^{+}, 78\right)$, $263.1\left([\mathrm{M}+\mathrm{Na}]^{+}, 100\right), 239.1\left([\mathrm{M}-\mathrm{H}]^{-}, 100\right), 274.9$ $\left(\left[\mathrm{M}+{ }^{35} \mathrm{Cl}\right]^{-}, 29\right)$.

\section{6-Bromoisatin-3Z-oxime (18m)}

Compound 18m $(0.16 \mathrm{~g}, 78 \%)$ was obtained as a yellow solid; $\mathrm{R}_{F}=0.15$ (silica gel, toluene $/ n$ heptane/ethyl acetate/formic acid, 80:20:10:3); $\mathrm{mp}=224-226^{\circ} \mathrm{C}$;

IR (KBr): $v=3384 s, 3220 s, 3178 s, 2900 m, 2864 m$, $1726 \mathrm{vs}, 1616 \mathrm{vs}, 1508 \mathrm{~m}, 1474 \mathrm{~m}, 1438 \mathrm{~s}, 1372 \mathrm{~m}$, $1336 m, 1294 w, 1278 w, 1244 w, 1212 w, 1188 w$, $1112 w, 1062 w, 1018 s, 860 m, 816 m, 664 m \mathrm{~cm}^{-1}$; UV-vis $(\mathrm{MeOH}): \lambda(\log \varepsilon)=261$ (4.29), 297 (3.00), $364(3.29) \mathrm{nm}$;

${ }^{1} \mathrm{H}$ NMR (400 MHz, DMSO-d 6$): \delta=13.47(s, 1 \mathrm{H}$, $\mathrm{NOH}), 10.81(s, 1 \mathrm{H}, \mathrm{NH}), 7.85(d, J=8.1 \mathrm{~Hz}, 1 \mathrm{H}, 4-$ $\mathrm{H}), 7.21(d, J=8.1 \mathrm{~Hz}, 1 \mathrm{H}, 5-\mathrm{H}), 7.02(s, 1 \mathrm{H}, 7-\mathrm{H})$ ppm; ${ }^{13} \mathrm{C}$ NMR (125 MHz, DMSO-d 6 ): $\delta=164.2(\mathrm{C}-$ 2), 144.9 (C-3), 143.4 (C-7a), 128.4 (C-6), 124.8 (C4), 124.7 (C-5), 114.9 (C-3a), 113.1 (C-7) ppm;

MS (ESI, MeOH): $\mathrm{m} / \mathrm{z}(\%)=243.1\left(\left[{ }^{81} \mathrm{M}+\mathrm{H}\right]^{+}, 78\right)$, $265.0\left(\left[{ }^{81} \mathrm{M}+\mathrm{Na}\right]^{+}, 100\right), 504.7\left(\left[2^{81} \mathrm{M}+\mathrm{Na}\right]^{+}, 49\right)$, $239.0\left(\left[{ }^{79} \mathrm{M}-\mathrm{H}\right]^{-}, 100\right), 502.7\left(\left[2^{79} \mathrm{M}-2 \mathrm{H}+\mathrm{Na}\right]^{-}, 5\right)$; analysis calcd for $\mathrm{C}_{8} \mathrm{H}_{5} \mathrm{BrN}_{2} \mathrm{O}_{2}$ (241.04): C 39.86, $\mathrm{H}$ 2.09, N 11.62; found C 39.64, H 2.21, N 11.37.

\section{7-Bromoisatin-3Z-oxime (18n)}

Compound 18n (0.44 g, 82\%) was obtained as a yellow solid; $\mathrm{R}_{F}=0.20$ (silica gel, toluene/nheptane/ethyl acetate/formic acid, 80:20:10:3); $\mathrm{mp}=260-264^{\circ} \mathrm{C}$;

IR (KBr): $v=3178 s, 3156 s, 3072 s, 2892 m, 2878 m$, $2790 m, 1732 v s, 1634 m, 1616 v s, 1580 m, 1472 m$, $1454 m, 1428 s, 1342 s, 1294 w, 1224 m, 1172 s, 1134 m$, $1044 \mathrm{~s}, 816 \mathrm{~m}, 790 \mathrm{~m}, 728 \mathrm{~m}, 690 \mathrm{~s} \mathrm{~cm}^{-1}$;

UV-vis $(\mathrm{MeOH}): \lambda(\log \varepsilon)=227$ (3.49), 244 (3.49), 256 (4.19), 298 (3.49), 372 (3.49) nm;

${ }^{1} \mathrm{H}$ NMR (400 MHz, DMSO-d 6$): \delta=13.56(s, 1 \mathrm{H}$, $\mathrm{NOH}), 10.98(s, 1 \mathrm{H}, \mathrm{NH}), 7.95(d, J=7.4 \mathrm{~Hz}, 1 \mathrm{H}, 6-$ $\mathrm{H}), 7.55(d, J=8.2 \mathrm{~Hz}, 1 \mathrm{H}, 4-\mathrm{H}), 6.98(d d, J=8.2$, $7.5 \mathrm{~Hz}, 1 \mathrm{H}, 5-\mathrm{H}) \mathrm{ppm} ;{ }^{13} \mathrm{C}$ NMR $(100 \mathrm{MHz}$, DMSO$\left.\mathrm{d}_{6}\right): \delta=164.2(\mathrm{C}-2), 143.8$ (C-3), 141.7 (C-7a), 134.5 (C-6), 125.9 (C-4), 123.7 (C-5), 117.5 (C-3a), 102.6 (C-7) ppm;

MS (ESI, $\mathrm{MeOH}): \mathrm{m} / \mathrm{z}(\%)=241.1\left(\left[{ }^{79} \mathrm{M}+\mathrm{H}\right]^{+}, 100\right)$, $257.9\left(\left[{ }^{79} \mathrm{M}+\mathrm{NH}_{4}\right]^{+}, 99\right), 263.0\left(\left[{ }^{79} \mathrm{M}+\mathrm{Na}\right]^{+}, 96\right)$, $294.6\left(\left[{ }^{79} \mathrm{M}+\mathrm{Na}+\mathrm{MeOH}\right]^{+}, \quad 25\right), 239.0 \quad\left(\left[{ }^{79} \mathrm{M}-\mathrm{H}\right]^{-}\right.$, 100);

analysis calcd for $\mathrm{C}_{8} \mathrm{H}_{5} \mathrm{BrN}_{2} \mathrm{O}_{2}$ (241.04): C 39.86, H 2.09, N 11.62; found C 39.51, H 1.96, N 11.42.

\section{Molecular Modelling}

Crystal structure of the ee $\mathrm{AChE}(\mathrm{PDB}=1 \mathrm{C} 2 \mathrm{O})$ was retrieved from protein databank (rscb.org). The enzyme was prepared according to usual procedures. Polar hydrogen atoms were added, water molecules removed, and Gasteiger charges were added. The Ligand minimisation and preparation was performed with MMFF94 force field in Datawarrior. Open Babel was used creating the pdbqt files for Autodock. Calculations were performed with Autodock $4{ }^{36}$. Grid Center: 35.180, 72.374, -87.078, Grid Points 126, 126, 126 with 0.225 Angstroms spacing. Lamarckian genetic algorithm with standard GA parameters: population size $=250$; number of evaluations $=$ 25000000; number of generations 27000; GA runs = 10. Analysis of the docking poses was done with MGLTools 1.5.6; figures were created with PyMOL.

\section{Acknowledgements}

Many thanks are due to A. Manthai for experimental help and the preparation of several of the compounds, to Dr. D. Ströhl and his team for the NMR spectra and the late Dr. R. Kluge for the MS-measurements. IRand UV-vis spectra were recorded by V. Simon. Additional biological investigations were performed by Dr. L. Fischer, and we thank Dr. Th. Müller (Dep. of Haematology/Oncology, Martin-Luther Universität Halle-Wittenberg) for providing the cell lines. JMG was supported by a $\mathrm{PhD}$ grant from "Stiftung der Deutschen Wirtschaft". The authors declare no conflict of interests.

\section{References}

1- I. Łozińska, A. Świerczyńska, Z. Molęda, A. M. Hartman, A. K. H. Hirsch, Z. Czarnocki, Donepezilmelatonin hybrids as butyrylcholinesterase inhibitors: Improving binding affinity through varying mode of linking fragments, Arch. Pharm., 2018, 1800194.

2- P. Costanzo, L. Cariati, D. Desiderio, R. Sgammato, A. Lamberti, R. Arcone, R. Salerno, M. Nardi, M. Masullo, M. Oliverio, Design, Synthesis, and Evaluation of Donepezil-Like Compounds as AChE and BACE-1 Inhibitors, ACS Med. Chem. Lett., 2016, 7, 470-475.

3- K. O. Yerdelen, M. Koca, B. Anil, H. Sevindik, Z. Kasap, Z. Halici, K. Turkaydin, G. Gunesacar, Synthesis of donepezil-based multifunctional agents for the treatment of Alzheimer's disease, Bioorg. Med. Chem. Lett., 2015, 25, 5576-5582.

4- M. Koca, K. O. Yerdelen, B. Anil, Z. Kasap, H. Sevindik, I. Ozyurek, G. Gunesacar, K. Turkaydin, Design, synthesis and biological activity of $1 \mathrm{H}$-indene2-carboxamides as multi-targeted anti-Alzheimer agents, J. Enzyme Inhib. Med. Chem., 2016, 31, 13-23.

5- M. S. Altowyan, M. Ali, S. M. Soliman, A. M. AlMajid, M. S. Islam, S, Yousuf, M. I. Choudhary, H. A. Ghabbour, A. Barakat, Synthesis, computational studies and biological activity of oxamohydrazide 
derivatives bearing isatin and ferrocene scaffolds, $\mathbf{J}$. Mol. Struct., 2020, 1202, 127372.

6- A. V. Bogdanov, I. F. Zaripova, A. D. Voloshina, A. S. Sapunova, N. V. Kulik, I. V. Tsivunina, A. B. Dorbrynin, V. F. Mironov, J. Fluorine Chem., 2019, 227, 109345.

7- M. M. H. Arief, W. I. A. El-Dougdoug, M. A. Sayed, Synthesis of some new isatin derivatives of expected biological activities, J. Basic Environm. Sci., 2019, 6, 149-155.

8- T. Sandmeyer, Über Isonitrosoacetanilide und deren Kondensation zu Isatinen, Helv. Chim. Acta, 1919, 2, 234-242.

9- Y. S. Tingare, M. T. Shen, C. Su, S. Y. Ho, S. H. Tsai, B. -R. Chen, W. R. Li, Novel Oxindole Based Sensitizers: Synthesis and Application in DyeSensitized Solar Cells, Org. Lett., 2013, 15, 4292-4295.

10- J. B. Campbell, E. J. Warawa, Preparation and formulation of heterocyclic fused tricyclic compounds as anxiolytics, EP245053A119871111.

11- L. -S. Feng, M. -L. Liu, S. Zhang, Y. Chai, B. Wang, Y.-B. Zhang, K. Lv, Y. Guan, H. -Y. Guo, C. -L. Xiao, Synthesis and in vitro antimycobacterial activity of $8-\mathrm{OCH}_{3}$ ciprofloxacin methylene and ethylene isatin derivatives, Eur. J. Med. Chem., 2011, 46, 341-348.

12- Z. Wang, C. Wang, Y. Sun, N. Zhang, Z. Liu, J. Liu, A novel strategy to the synthesis of 4-anilinoquinazoline derivatives, Tetrahedron, 2014, 70, 906-913.

13- P. Polychronopoulos, P. Magiatis, A. L. Skaltsounis, V. Myrianthopoulos, E. Mikros, A. Tarricone, A. Musacchio, S.M. Roe, L. Pearl, M. Leost, P. Greengard, L. Meijer, Structural Basis for the Synthesis of Indirubins as Potent and Selective Inhibitors of Glycogen Synthase Kinase-3 and CyclinDependent Kinases, J. Med. Chem., 2004, 47, 935-946.

14- G. L. Ellman, K. D. Courtney, V. J. Andres, R. M. Featherstone, A new and rapid colorimetric determination of acetylcholinesterase activity, Biochem. Pharmacol., 1961, 7, 88-95.

15- A. Loesche, A. Kowitsch, R. Csuk, S. D. Lucas, Z. Al-Halabi, W. Sippl, A. Al-Harrasi, Ursolic and oleanolic acid derivatives with cholinesterase inhibiting potential, Bioorg. Chem., 2018, 85, 23-32.

16- S. Liu, W. Wei, Y. Li, X. Liu, X. Cao, K. Lei, M. Zhou, Design, synthesis, biological evaluation and molecular docking studies of phenylpropanoid derivatives as potent anti-hepatitis B virus agents, Eur. J. Med. Chem., 2015, 95, 473-482.

17- Suresh, D. Kumar, J. S. Sandhu, Bismuth(III) Chloride-Mediated, Efficient, Solvent-Free, MWIEnhanced Doebner Condensation for the Synthesis of (E)-Cinnamic Acids, Synth. Commun., 2010, 40, 1915-1919.

18- T. Takahashi, M. Miyazawa, Tyrosinase inhibitory activities of cinnamic acid analogues, Pharmazie, 2010, 65, 913-918.

19- K. Kindler, K. Lührs, Studien über den Mechanismus chemischer Reaktionen, XXII. (Über Hydrierungen und über spezifische Hydrierungen mittels gebundenen Wasserstoffs, VI). Hydrierungen mittels Terpenen, Ann. Chem., 1965, 685, 36-48.

20- J. Koo, The Synthesis of 4,5,6-Trimethoxyindene- and 4,5,6-Trimethoxyindanecarboxylic Acids and Esters, J. Am. Chem. Soc., 1953, 75, 1889-1891.
21- C. Pan, X. Zeng, Y. Guan, X. Jiang, L. Li, H. Zhang, Design and Synthesis of Brazilin-Like Compounds, Synlett, 2011, 425-429.

22- T. M. Harris, C. R. Hauser, Benzylation at the Terminal Methyl Group of Certain Unsymmetrical $\beta$-Diketones Through One of Two Possible Intermediate Dicarbanions, J. Am. Chem. Soc., 1959, 81, 1160-1164.

23- T. Dohi, N. Takenaga, A. Goto, H. Fujioka, Y. Kita, Clean and Efficient Benzylic C $-\mathrm{H}$ Oxidation in Water Using a Hypervalent Iodine Reagent: Activation of Polymeric Iodosobenzene with $\mathrm{KBr}$ in the Presence of Montmorillonite-K10, J. Org. Chem., 2008, 73, 73657368.

24- A. Romeo, H. Corrodi, E. Hardegger, Umsetzungen des o-Nitrophenylessigesters und des 2-Chlor-6-nitrophenyl-brenztraubensäureesters, Helv. Chim. Acta, 1955, 38, 463-467.

25- S. B. Kadin, Preparation and formulation of analgesic and antiinflammatory 1,3-diacyl-2-oxindole compounds, US4690943A19870901.

26- M. E. Matheus, F. D. A. Violante, S. J. Garden, A. C. Pinto, P. D. Fernandes, Isatins inhibit cyclooxygenase2 and inducible nitric oxide synthase in a mouse macrophage cell line, Eur. J. Pharmacol., 2007, 556, 200-206.

27- K. C. Joshi, V. N. Pathak, S. K. Jain, Studies of potential organo-fluorine antibacterial agents. Part 5: Synthesis and antibacterial activity of some new fluorine-containing indole-2,3-dione derivatives, Pharmazie, 1980, 35, 677-679.

28- B. R. Baker, R. E. Schaub, J. P. Joseph, F. J. McEvoy, J. H. Williams, An antimalarial alkaloid from hydrangea. XV. Synthesis of 5-, 6-, 7-, and 8-derivatives with two identical substituents, J. Org. Chem., 1952, 17, 149-156.

29- L. Ettinger, P. Friedländer, Über 6.6'-Dibromindirubin, Ber. Dt. Chem. Ges., 1912, 45, 2081-2083.

30- S. Inagaki, Diphenylisatin and its derivatives. IV. Monobromo derivatives of dianisoleisatin and its oxidation product, Yakugaku Zasshi, 1938, 58, 961-975.

31- A. Beauchard, H. Laborie, H. Rouillard, O. Lozach, Y. Ferandin, R. L. Guével, C. Guguen-Guillouzo, L. Meijer, T. Besson, V. Thiéry, Synthesis and kinase inhibitory activity of novel substituted indigoids, Bioorg. Med. Chem., 2009, 17, 6257-6263.

32- F. Ziegler, T. Kappe, R. Salvador, Synthesen von Heterocyclen, 44. Mitt.: Eine Synthese des Isatins, Monatsh. Chem., 1963, 94, 453-459.

33- C. A. Fetscher, M. T. Bogert, Researches on quinaolines. XLIV. The synthesis of some new quinazoline derivatives of veratrole akin to alkaloids, J. Org. Chem., 1939, 4, 71-87.

34- J. Gray, D. R. Waring, 3-Amino-2,1-benzoisothiazole. Synthesis of some chloro and trifluoromethyl derivatives, J. Heterocycl. Chem., 1980, 17, 65-67.

35- D. H. Klaubert, J. H. Sellstedt, C. J. Guinosso, R. J. Capetola, S. C. Bell, N-(Aminophenyl)oxamic acids and esters as potent, orally active antiallergy agents, J. Med. Chem., 1981, 24, 742-748.

36- G. M. Morris, R. Huey, W. Lindstrom, M. F. Sanner, R. K. Belew, D. S. Goodsell, A. J. Olson, AutoDock and AutoDockTools: Automated docking with selective receptor flexibility, J. Comput. Chem., 2009, $30,2785-2791$ 